\title{
Eliminating hypoxic tumor cells improves response to PARP inhibitors in homologous recombination- deficient cancer models
}

\author{
Manal Mehibel, ${ }^{1}$ Yu Xu, ${ }^{1}$ Caiyun G. Li, ${ }^{1}$ Eui Jung Moon, ${ }^{1}$ Kaushik N. Thakkar, ${ }^{1}$ Anh N. Diep, ${ }^{1}$ Ryan K. Kim, ${ }^{1}$ Joshua D. Bloomstein, ${ }^{1}$ \\ Yiren Xiao, ${ }^{1}$ Julien Bacal, ${ }^{2}$ Joshua C. Saldivar, ${ }^{2}$ Quynh-Thu Le, ${ }^{1}$ Karlene A. Cimprich, ${ }^{2}$ Erinn B. Rankin,, ${ }^{1,3}$ and Amato J. Giaccia ${ }^{1,4}$ \\ 'Division of Radiation and Cancer Biology, Department of Radiation Oncology, Stanford University Medical Center, Stanford, California, USA. ²Department of Chemical and Systems Biology, Stanford \\ University School of Medicine, Stanford, California, USA. ${ }^{3}$ Department of Obstetrics and Gynecology, Stanford University Medical Center, Stanford, California, USA. ${ }^{4}$ Oxford Institute for Radiation Oncology, \\ University of Oxford, Oxford, United Kingdom.
}

\begin{abstract}
Hypoxia, a hallmark feature of the tumor microenvironment, causes resistance to conventional chemotherapy, but was recently reported to synergize with poly(ADP-ribose) polymerase inhibitors (PARPis) in homologous recombination-proficient (HR-proficient) cells through suppression of HR. While this synergistic killing occurs under severe hypoxia (<0.5\% 0xygen), our study shows that moderate hypoxia ( $2 \%$ oxygen) instead promotes PARPi resistance in both HR-proficient and -deficient cancer cells. Mechanistically, we identify reduced ROS-induced DNA damage as the cause for the observed resistance. To determine the contribution of hypoxia to PARPi resistance in tumors, we used the hypoxic cytotoxin tirapazamine to selectively kill hypoxic tumor cells. We found that the selective elimination of hypoxic tumor cells led to a substantial antitumor response when used with PARPi compared with that in tumors treated with PARPi alone, without enhancing normal tissue toxicity. Since human breast cancers with BRAC1/2 mutations have an increased hypoxia signature and hypoxia reduces the efficacy of PARPi, then eliminating hypoxic tumor cells should enhance the efficacy of PARPi therapy.
\end{abstract}

\section{Introduction}

Cytotoxic therapies have long been the backbone of cancer therapy, as they represent an effective means of killing large numbers of tumor cells. A common problem with cytotoxic therapies is normal tissue toxicity, which often results in cancer patients being unable to receive sufficient drug concentrations to eradicate their tumors (1). Over the years, the directed application of cytotoxic therapies to tumors with mutations in genes that disrupt DNA damage signaling and repair pathways has demonstrated improved tumor control with acceptable levels of normal tissue toxicity (2). Therefore, targeting the DNA damage response is an attractive therapeutic strategy, and poly(ADP-ribose) polymerase inhibitors (PARPis) are still one of the best examples of this concept (3). The therapy works by inhibiting the function of the PARP enzyme in cancer cells to repair certain types of DNA damage (4). PARP1 protein, a well-recognized sensor of DNA damage, plays an important role in DNA repair and, specifically, DNA single-strand break (SSB) repair through the base excision repair (BER) pathway (5) as well as DNA double-strand break (DSB) repair by alternative nonhomologous end-joining (alt-NHEJ) (6, 7). Mechanistically, the catalytic function of the PARP1 enzyme is activated after it binds to the site of DNA damage, which triggers a series of allosteric changes in its structure (8). Activated PARP1

Conflict of interest: The authors have declared that no conflict of interest exists. Copyright: (5) 2021, American Society for Clinical Investigation.

Submitted: November 23, 2020; Accepted: April 21, 2021; Published: June 1, 2021.

Reference information: J Clin Invest. 2021;131(11):e146256.

https://doi.org/10.1172/JCl146256. is responsible for its own autoPARylation as well as PARylation and subsequent recruitment of other DNA repair proteins to the site of DNA damage. The negative charge from the PAR chains is suggested to be the mechanism by which PARP1 is released from DNA, allowing access for other repair enzymes to the lesion (4, 8). PARPis work by inhibiting the catalytic activity of PARP and/ or trapping the protein on DNA damage sites, thereby preventing the repair of SSBs, which results in the generation of lethal DSBs during replication (4).

PARPis exhibit clinical activity in tumors with BRCA mutations because deficiency in homologous recombination (HR) prevents tumor cells from efficiently repairing the deleterious DSBs accumulated during cell replication, leading to cell lethality $(9,10)$. Interestingly, HR deficiency is not restricted to tumors with BRCA mutations, as follow-up studies showed that disruption of other HRrelated genes, such as Fanconi anemia (11), ATM (12), or isocitrate dehydrogenase (IDH1/2) (13), renders tumors sensitive to PARPis.

Hypoxia, a hallmark feature of solid tumors, occurs as a consequence of the imbalance between rapid tumor growth and the poorly organized vasculature $(14,15)$. Oxygen levels within a tumor are heterogeneous and can range from moderate $(1 \%-2 \%$ $\left.\mathrm{O}_{2}\right)$ to severe $\left(<0.01 \% \mathrm{O}_{2}\right)(16)$. Additionally, hypoxia is an adverse clinical prognostic factor for patient outcome and correlates with tumor metastasis as well as resistance to conventional chemotherapy and radiotherapy (14). Hypoxia can be associated with HR repair abnormalities as a result of epigenetic silencing of BRCA1 (17), transcriptional suppression of BRCA1, BRCA2, and RAD51 genes (18-20), and functional inhibition of HR by the S-2hydroxyglutarate (S-2HG) metabolite (13). Through suppression 
of HR, hypoxia at oxygen levels below $0.5 \%$ confers sensitivity of HR-proficient tumors to PARPi, thereby inducing synthetic lethality $(21,22)$. The role of hypoxia in HR-deficient tumors is yet to be investigated, and as a unique feature of solid tumors that is not present in normal tissues, hypoxia is considered an exploitable target in cancer therapy (14).

Studies have shown that the clinical efficacy of PARPis in BRCA-mutant ovarian and breast cancers is highly promising (23, $24)$. Although response rates were limited to $30 \%-40 \%$, which is significantly superior to the expected rates of $20 \%$ or lower achieved with conventional cytotoxic chemotherapies $(25,26)$, they still fall short of the observed $50 \%-70 \%$ response rates found with other targeted therapies. In an era of personalized therapy, a lower than $50 \%$ response rate to a targeted therapy is considered unsatisfactory (27). This highlights our incomplete understanding of the complexity of DNA-repair pathways in the context of the tumor microenvironment and the necessity of investigating the mechanisms by which tumors harness resistance to PARP inhibition, especially in HR-deficient tumors. In the current study, we explore the effect of different levels of hypoxia on the response of HR-deficient tumors to PARPi with the view of developing strategies that could potentially enhance their clinical efficacy.

\section{Results}

Hypoxia causes resistance to PARPi in an HIF-independent pathway. We set out to investigate the effect of hypoxia on sensitivity of HR-deficient cells to PARPi. To test this, we exposed a panel of HR-deficient cell lines, SUM149 (BRCA1 mutation), OVCAR8 (functional deficiency of BRCA1 gene), CAPAN1 (BRCA2 mutation), and HT1080 (heterozygous IDH1 R132C mutation), to PARPis (olaparib and talazoparib) under normoxic or moderate hypoxic ( $2 \%$ oxygen) conditions. Our results showed that cells cultured in hypoxia were significantly more resistant to PARPi treatment independently of the type of HR deficiency (Figure 1A and Supplemental Figure 1A; supplemental material available online with this article; https://doi.org/10.1172/JCI146256DS1). For example, there was substantial resistance of the hypoxic SUM149 cells to olaparib and talazoparib, with about 8-fold and 4-fold increases in cell survival compared with normoxic cells at doses of $1 \mu \mathrm{M}$ and $1 \mathrm{nM}$, respectively. In HT1080 cells, we observed a 6-fold and 13-fold increase in survival of hypoxic versus normoxic cells treated with $1 \mu \mathrm{M}$ olaparib and $10 \mathrm{nM}$ talazoparib, respectively.

We also found that survival of the HR-proficient MDA231 cells was significantly increased in hypoxia ( $2 \%$ oxygen) compared with normoxia, although higher doses of PARPi were required to observe measurable cellular toxicity in normoxia (Figure 1A). Sensitivity of these cells to PARPi was instead significantly enhanced under conditions of severe hypoxia (0.5\% oxygen), consistent with previous reports (Figure 1B) $(18,19,21,22)$. Analysis of HR gene expression in MDA231 cells indicated that BRCA1 and RAD51 gene expression was significantly suppressed under $0.5 \%$, but not under $2 \%$, oxygen conditions (Figure $1 \mathrm{C}$ ).

PARP1 is responsible for more than $90 \%$ of cellular PARylation (28) and its loss or reduced expression in human cancer cell lines results from PARPi treatment $(12,29)$. In our experiments, PARP1 protein levels detected by Western blotting in SUM149 cells treated with either olaparib or talazoparib remained unal- tered under hypoxia (Supplemental Figure 1B). In addition, both PARPis were able to efficiently suppress PARylation in a range of hypoxic cells, ruling out loss of PARP1 protein, compensation by other PARP family proteins, loss of PAR glycohydrolase (PARG), or upregulation of any drug efflux transporters as the cause of the observed resistance (Supplemental Figure 1, C and D) (30-32).

There were also no significant changes in cell-cycle distribution (Supplemental Figure 1, E and F) or proliferation (Supplemental Figure $1 G$ ) upon incubation under hypoxia, which strongly excludes the possibility of a confounding effect arising from altered cell-cycle distribution. This is in accordance with previous studies that showed that moderate hypoxia does not affect cellcycle distribution or cellular proliferation, suggesting cell-cycle perturbations are not involved in PARP resistance under 2\% oxygen conditions $(20,33)$.

We further tested the effect of different moderate oxygen tensions on OVCAR8 cells treated with PARPis. Response to PARPi was oxygen dependent, with cells maintained under $5 \%$ oxygen levels showing more drug resistance than normoxic cells, but more drug sensitivity than cells cultured under $2 \%$ oxygen conditions (Supplemental Figure 1H). To investigate whether the observed hypoxia-mediated resistance to PARPi is HIF dependent, we generated HIF-deficient OVCAR8 cells by knocking down the different HIF components (HIF1 $\alpha$, HIF $2 \alpha$, and HIF $\beta$ ) and then tested their sensitivity to PARPi under normoxic and hypoxic conditions. HIF1 and HIF2 knockdown did not alter cellular sensitivity to PARPi under either oxygen tensions (Figure 1D), suggesting that HIF is not a mediator of the observed cellular resistance to PARPi.

Hypoxia is associated with resistance to PARPi in vivo. We aimed to test whether our observation of hypoxia-mediated resistance to PARPis in HR-deficient cells also occurs in tumors. OVCAR8 xenografts were treated once daily with $50 \mathrm{mg} / \mathrm{kg}$ olaparib or vehicle and assayed for DNA damage-induced apoptosis (Schema shown in Figure 2A). Tumor lysates were used to confirm that inhibition of PARP activity was achieved in vivo through a reduction in PAR levels, and this was associated with an overall increase in tumor DNA damage as detected by $\gamma \mathrm{H} 2 \mathrm{AX}$ (Figure 2B). In support of hypoxia-induced resistance to PARPis in cell culture, we found decreased TUNEL-positive cells in hypoxic (pimonidazole or CA9 positive) subregions of PARPi-treated tumors as compared with normoxic regions by immunohistochemical staining, confirming that hypoxia is associated with resistance to PARPi-induced damage in vivo (Figure $2 \mathrm{C}$ ).

The efficacy of olaparib in a range of breast cancer patientderived tumor xenografts (PDTXs) was previously tested by Bruna et al. (34) and the results archived in the Breast Cancer PDTX Encyclopaedia biobank (http://caldaslab.cruk.cam.ac.uk/bcape). We applied a previously validated, robust hypoxia signature, developed by Buffa et al. (35), and observed a strong inverse correlation between hypoxia scores of the tumors and their sensitivity to olaparib therapy. PDTXs with higher hypoxic scores were the most resistant to PARPi therapy based on changes in tumor growth (Figure 2D). Thus, both tumor cell-derived xenografts and PDTXs indicate that hypoxia was linked with decreased PARPi efficacy.

Hypoxia is a common feature of cancers (15). By taking advantage of the expanded The Cancer Genome Atlas (TCGA) data to calculate the hypoxic score of different cancer types, we confirmed 
A

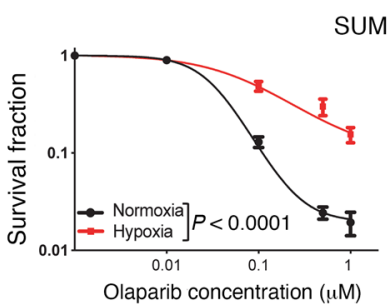

SUM149

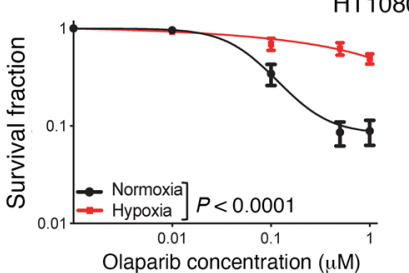

HT1080
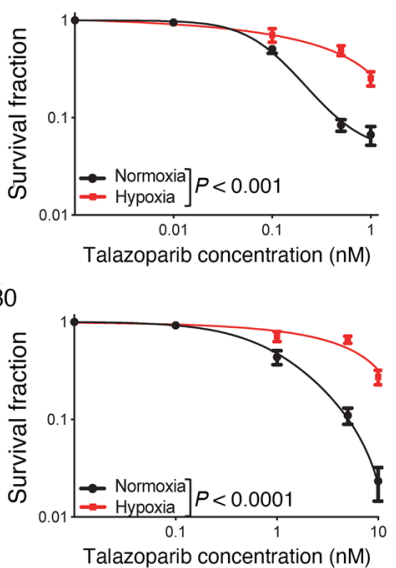

OVCAR8
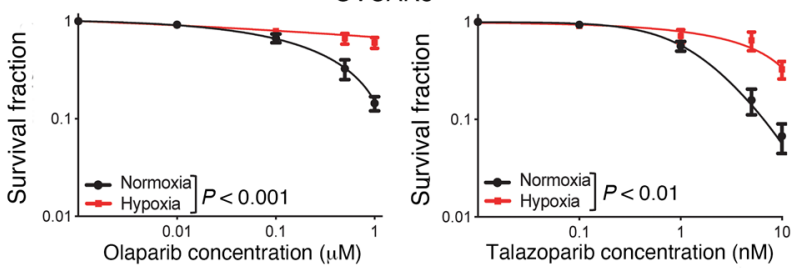

CAPAN 1
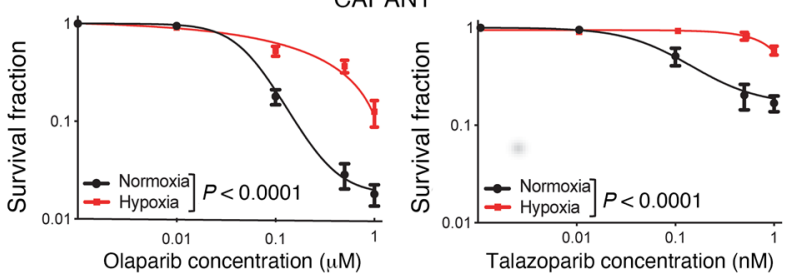

MDA231
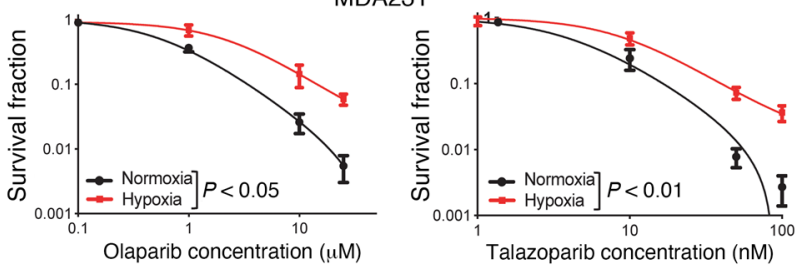

B
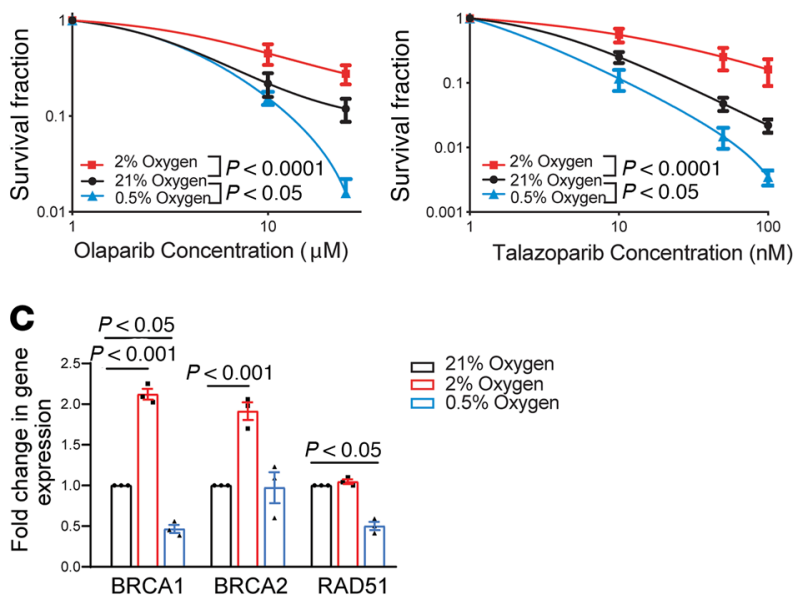

D
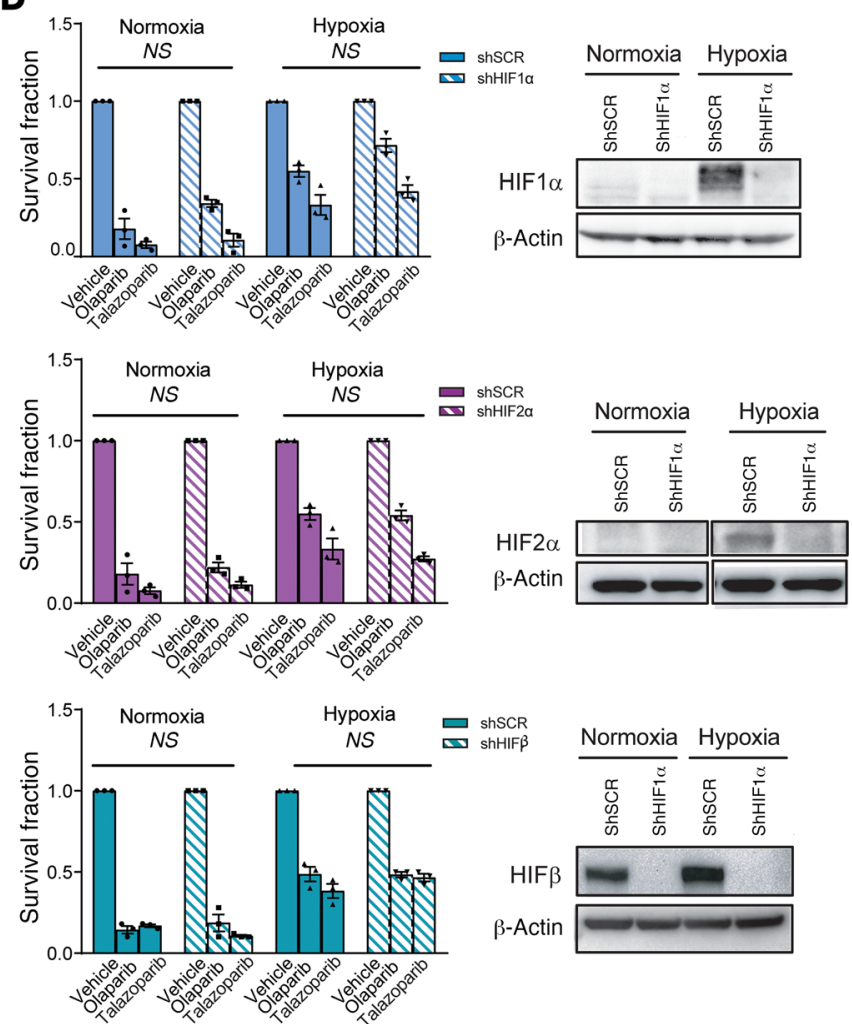

Figure 1. Hypoxia causes resistance to PARPis in an HIF-independent pathway. (A) Clonogenic formation of HR-deficient cells (SUM149, OVCAR, CAPAN-1, HT1080), and HR-proficient cells (MDA231) treated with indicated doses of olaparib or talazoparib for 7 days under normoxic (black lines) or hypoxic (red lines) conditions, followed by 7- to 10-day culture in the absence of inhibitor. Survival relative to vehicle-treated cells is plotted (2-way ANOVA, interaction $P$ value, normoxia versus hypoxia; at least 3 biological replicates). (B) Clonogenic formation of MDA231 cells treated with indicated doses of olaparib or talazoparib for 2 days under normoxia (black lines), $2 \%$ oxygen (red lines) or $0.5 \%$ oxygen (blue lines), followed by 7 - to 10 -day culture in the absence of inhibitor. Survival relative to vehicle-treated cells is plotted (2-way ANOVA, adjusted $P$ value, normoxia versus hypoxia; at least 3 biological replicates). See also Supplemental Figure 1B. (C) qRT-PCR measuring BRCA1, BRCA2, and RAD51 mRNA levels in MDA231 cells incubated under different oxygen tensions. Measurements were normalized to 185 mRNA levels and expressed as fold change compared with normoxia (2-way ANOVA, adjusted $P$ value; hypoxia versus normoxia for each gene; $n=3$ ). (D) Clonogenic survival assay of OVCAR8 cells expressing the indicated shRNA treated with olaparib or talazoparib for 7 days under normoxia or hypoxia. Interaction $P$ value by 2-way ANOVA shScr versus shHIF for each treatment, $n=3$ (left panels). Immunoblots (right panels) showing silencing efficiencies of shRNAs; $\beta$-actin is a loading control. See also Supplemental Figure $1 \mathrm{H}$. Data are represented as mean \pm SEM (represented by error bars). 
$\mathbf{A}$

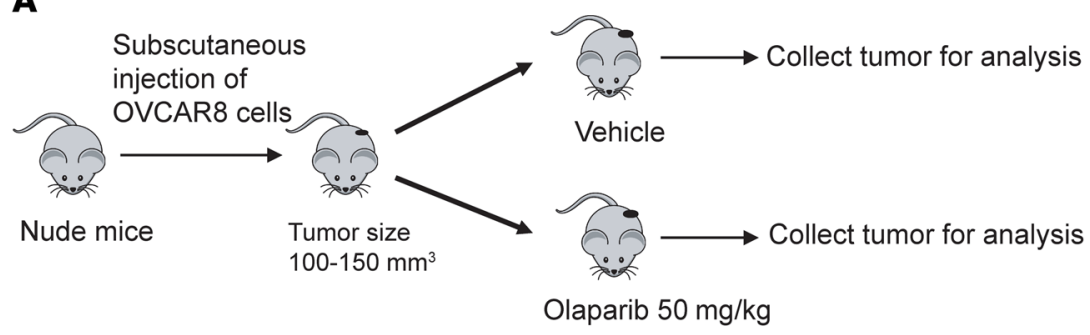

B

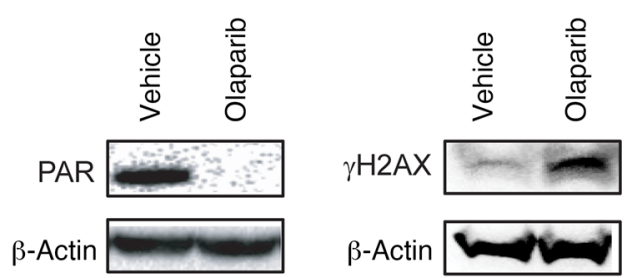

C
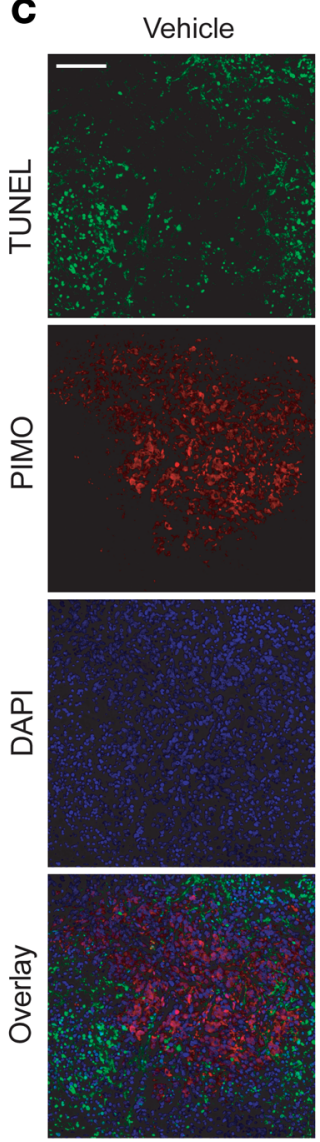

Olaparib
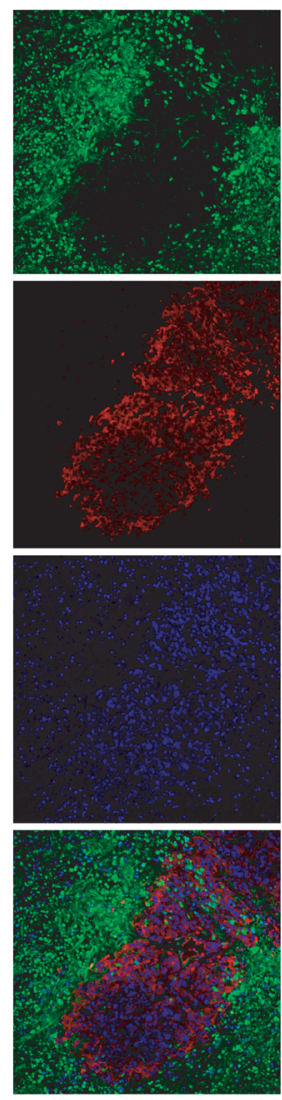
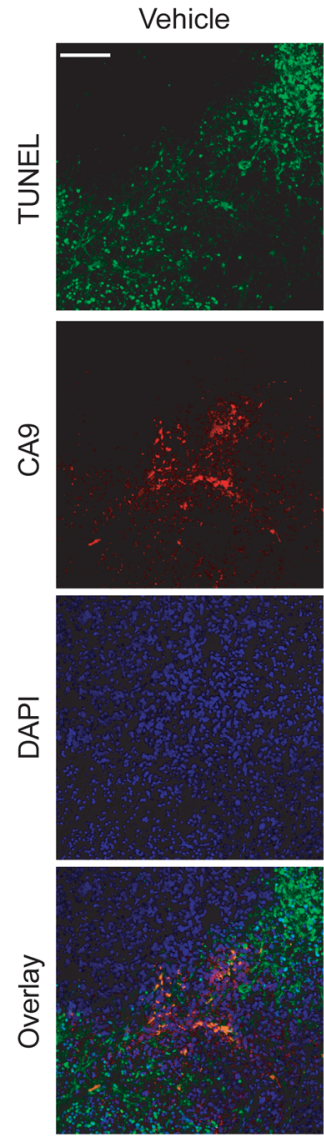

Olaparib
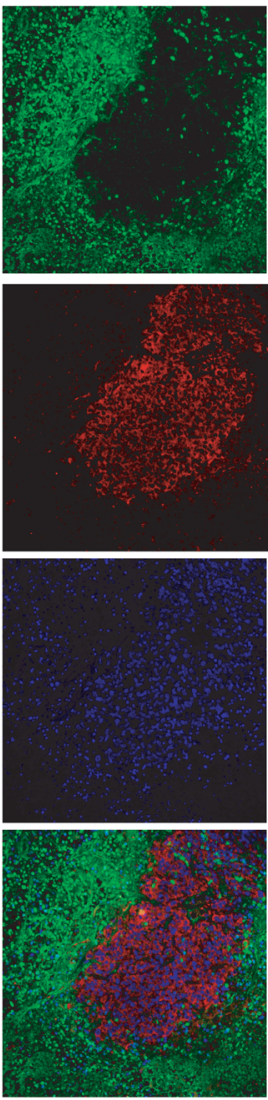

Figure 2. Hypoxia is associated with resistance to PARPi in vivo. (A) Schematic diagram of olaparib treatment. OVCAR8 xenografts were treated once daily for 2 days with vehicle or olaparib $(50 \mathrm{mg} / \mathrm{kg}$ ) and then collected for immunohistochemical staining and determination of PARP activity (vehicle, $n=3$; olaparib, $n=3$ ). (B) Western blot analysis of PAR levels (left panel) and $\gamma \mathrm{H} 2 \mathrm{AX}$ (right panel) in tumor lysates collected 2 hours after the final olaparib dose. $\beta$-Actin is a loading control. (C) Representative immunohistochemical staining of vehicle- and olaparib-treated tumors shows absence of TUNEL staining in pimonidazole-positive (left panel) and CA9-positive (right panel) regions. Scale bars: $100 \mu \mathrm{m}$. (D) Plot of relationship between hypoxia score of PDX tumors and their sensitivity to olaparib $(50 \mathrm{mg} / \mathrm{kg}) . R^{2}$ $=0.59, P=0.015$, Spearman's correlation.
D

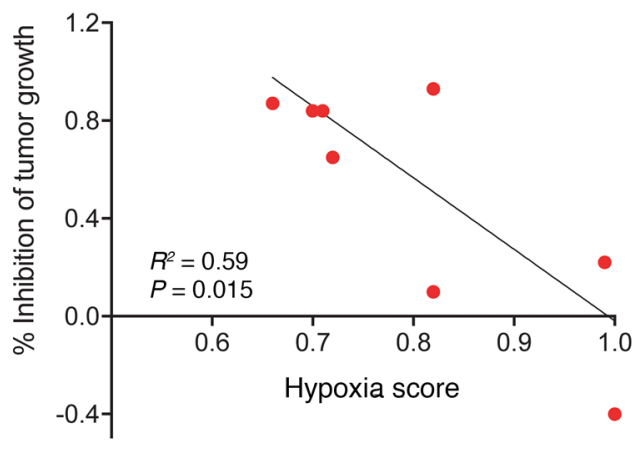

that hypoxia is present in a wide range of cancers (Supplemental Figure 2A.) Focusing on breast cancer, we showed that breast tumors are significantly more hypoxic than normal breast tissue, and more interestingly, breast tumors with BRCA1/2 mutations had significantly higher hypoxic scores when compared with non-
BRCA1/2-deficient tumors (Supplemental Figure 2B), a finding that, as far as we are aware, has not been previously recognized.

PARPi-induced DNA damage and activation of C-NHEJpathway are diminished in hypoxia. Treatment with PARPi for 48 hours in normoxia was associated with increased phosphorylation of DNA 
damage response (DDR) markers H2AX on serine 139 ( $\gamma \mathrm{H} 2 \mathrm{AX})$, KAP1 on serine 824 , and Chk1 on serine 345 , while phosphorylation of these proteins was significantly lower in PARPi-treated hypoxic cells (Figure 3A and Supplemental Figure 3A). Quantitative studies of DNA damage in SUM149, OVCAR8, and HT1080 cells revealed substantially increased $\gamma \mathrm{H} 2 \mathrm{AX}$ foci in normoxia as compared with hypoxia upon inhibition of PARP (Figures $3 \mathrm{~B}$ and Supplemental Figure 3B). Additionally, PARPi resulted in an augmentation of chromatin-bound PARP1 levels in normoxia versus hypoxia (Figure 3C) without affecting total cellular PARP1 levels (Supplemental Figure 1B), confirming that DNA damage occurs at higher levels in normoxia, since persistent PARP1-DNA complexes, induced by PARPi, are toxic to the cells (12).

Classical NHEJ (C-NHEJ), a highly error-prone repair process, is essential for effective toxicity of PARPi, and inhibition or downregulation of its components has been shown to diminish toxicity of PARPi in HR-deficient cells (36). Since this conclusion is based on cells treated under normoxic conditions, we sought to evaluate the role of the C-NHEJ pathway under hypoxic conditions. We examined the phosphorylation of DNA-dependent protein kinase, catalytic subunit (DNA-PKcs) on T2609, which is necessary for efficient C-NHEJ (37), and found that phosphorylation of this site in PARPi-treated cells was significantly higher under normoxia than hypoxia (Figure 3D and Supplemental Figure 3C). Coadministration of the DNA-PK inhibitor (DNA-PKi) NU7441 reversed the cytotoxic effects of PARPi in SUM149 cells under both culture conditions, suggesting that the C-NHEJ plays a crucial role for PARPi toxicity, but does not play a special role under hypoxic conditions (Figure 3E).

We further analyzed SUM149 cells for foci containing p53BP1 that is phosphorylated on S25. The location of S25 in the S/T-Q motif of p53BP1 is important for RAP1-interacting factor 1 (RIF1) binding, inhibiting resection and subsequent activation of the C-NHEJ pathway (38-40). Treatment with PARPi under hypoxia inhibited the induction of p53BP1 S25 phosphorylation and RIF1 foci as well as chromatin-bound p53BP1 protein, but not in cells treated with PARPi in normoxia (Figure $3, \mathrm{~F}-\mathrm{H}$ ). In addition, similar differences in 53BP1 foci between normoxia and hypoxia were also observed in PARPi-treated OVCAR8 and HT1080 cells (Supplemental Figure 3D).

Loss of 53BP1 and RIF1 correlate with reversion of HR deficiency, leading to alleviation of hypersensitivity to PARP inhibition (41-43). Although, in our study, the 2 proteins were lost to a certain extent in hypoxia, we failed to see a reversion of HR based on the fact that mRNA levels of BRCA1, BRCA2, and RAD51 genes remained suppressed (Supplemental Figure 3E). To corroborate this finding, we used the traffic light reporter (TLR) system that is designed to generate a flow-cytometric readout for repair at I-SceI-induced DNA DSBs (44). We found that resolution of these breaks through HR was unchanged because HR was already impaired, but that there was a significant reduction in the frequency of end-joining events in hypoxia (Figure 3I and Supplemental Figure $3 \mathrm{~F}$ ). This is consistent with the concept that PARPi-induced toxicity is reliant on activation of the C-NHEJ pathway.

Hypoxia-mediated PAPRi resistance is associated with low ROS production. Oxidative damage from ROS causes DNA damage in the form of base damage, SSBs, and DSBs (45), and PARPis rely on unrepaired DNA damage to cause cellular toxicity (4). We hypothesized that the lack of ROS in hypoxia is responsible for the observed differences in toxicity between normoxic and hypoxic cells. We found that production of ROS was significantly reduced after 48 hours of incubation in hypoxia as detected by dichlorofluorescein (DCF) fluorescence (Figure 4A and Supplemental Figure $4 \mathrm{~A})$, and this was associated with substantially reduced levels of 8 oxo-dG staining (Figure $4 \mathrm{~B}$ ). We then sought to determine whether hypoxic cells (SUM149 and HT1080) are intrinsically resistant to PARPi-induced DNA damage by measuring accumulation of $\gamma \mathrm{H} 2 \mathrm{AX}$ foci over time (Figures $4 \mathrm{C}$ and Supplemental Figure 4B). Upon treatment with PARPi, $\gamma \mathrm{H} 2 \mathrm{AX}$ was similarly induced in normoxia and hypoxia starting from 2 hours and continuing for 6 hours of drug incubation. However, at 24 hours of incubation, there were substantially higher levels of $\gamma \mathrm{H} 2 \mathrm{AX}$ foci in normoxia which remained elevated over 48 hours of treatment. Preincubation of cells for 48 hours in hypoxia prior to a 6-hour treatment with PARPi resulted in reduced induction of $\gamma \mathrm{H} 2 \mathrm{AX}$ foci (Figure 4D and Supplemental Figure 4C). Further treatment of these cells with $50 \mu \mathrm{M} \mathrm{H}_{2} \mathrm{O}_{2}$ for 2 hours in hypoxia reversed this effect in PARPi-treated cells (Figure 4E and Supplemental Figure 4D), suggesting that PARPi-induced damage can be enhanced in hypoxia with hydroxyl radical-generating stress.

Replication stress is often associated with the formation of DNA breaks (46). Previous findings by Maya-Mendoza and colleagues suggest that inhibition of PARylation induces replication stress and also leads to accelerated fork velocity, reduced cell viability, and increased genomic instability. These observations were, however, limited to normoxic culture conditions (47). We performed single-molecule DNA fiber analysis of replication fork progression in SUM149 cells treated with PARPi under normoxic as well as hypoxic conditions. After treatment with talazoparib, cells were pulsed with iododeoxyuridine (IdU) and then with chlorodeoxyuridine (CldU) to label newly synthesized DNA. The double labeling with IdU and CldU allowed the assessment of fork velocity by measuring the lengths of the CldU tracts (Figure 4F). In our experimental setting, talazoparib caused a substantial increase in fork velocity under normoxic and hypoxic conditions (Figure 4G).

Hypoxia is also implicated in resistance to other inhibitors of the alt-NHEJ pathway. Since PARP is involved in the repair of DNA DSBs through the alt-NHEJ pathway (6) and BRCA1/2-deficient

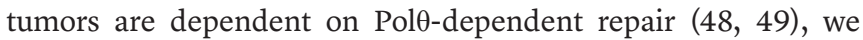
sought to investigate the hypoxic response to inhibition of elements of this pathway other than PARP.

We first investigated the lethality of L67, a competitive inhibitor that specifically targets DNA repair by inhibiting alt-NHEJ ligases (LigI/III) without affecting C-NHEJ, under normoxic and hypoxic conditions (50). Hypoxic SUM149, OVCAR8 cells, and HT1080 cells were substantially more resistant to L67 when compared with normoxic cells with a difference of up to 100-fold when the drug was used at a concentration of $6 \mu \mathrm{M}$ (Figure 5A). In addition, L67 reduced clonogenicity of cells treated with PARPi for 4 days only under normoxic conditions, which is in agreement with previously published data showing that leukemia cells were hypersensitive to the combination of L67 and PARPi (51). The combination, however, had no effect on PARPi-induced toxicity under hypoxia in our study (Figure 5, B and C). 
A

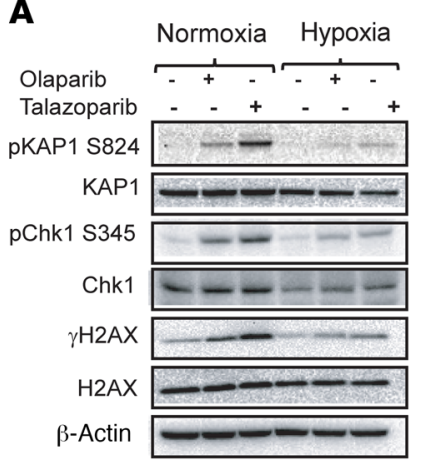

B
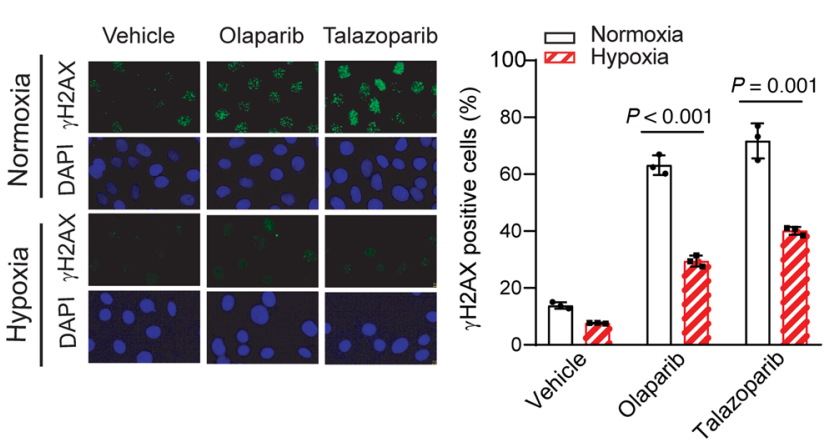

C

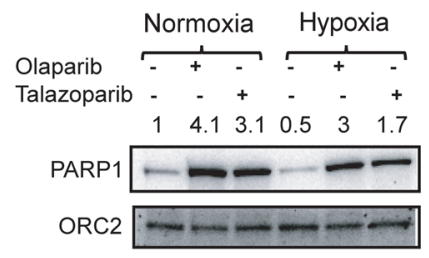

D

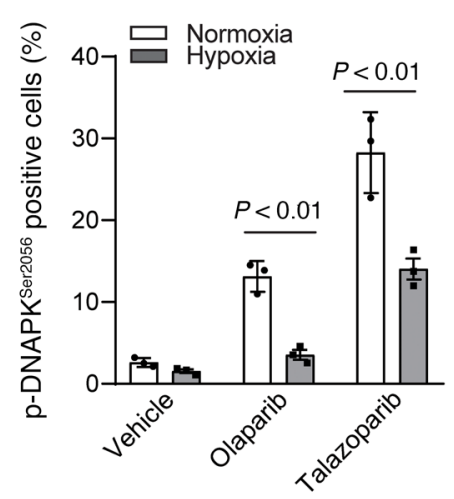

$\mathbf{F}$

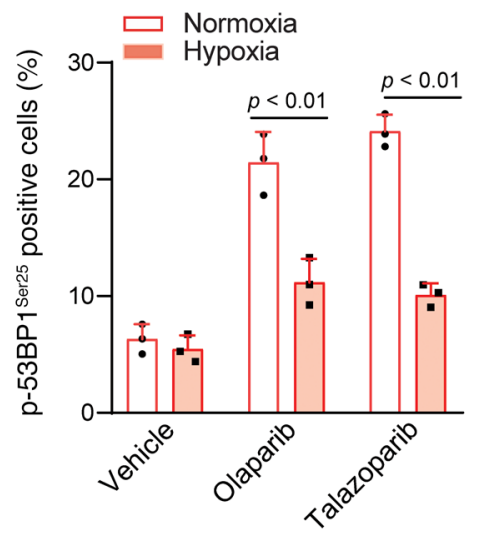

E
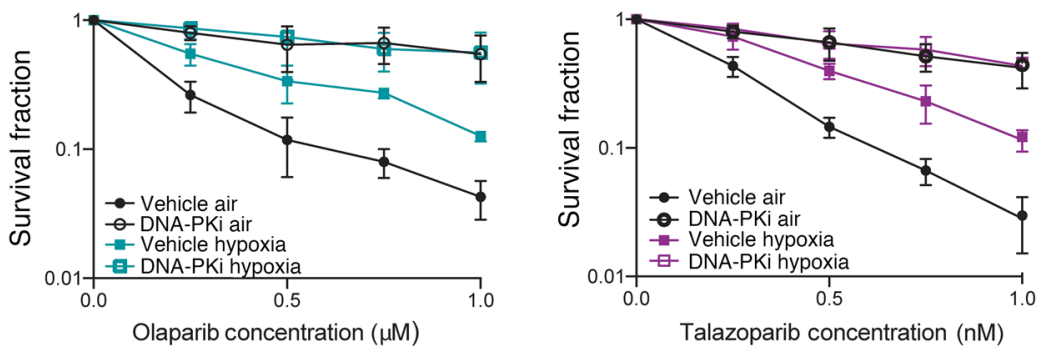

G

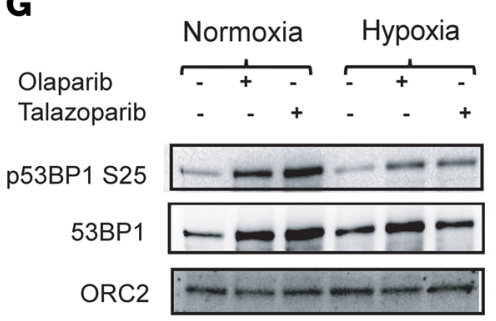

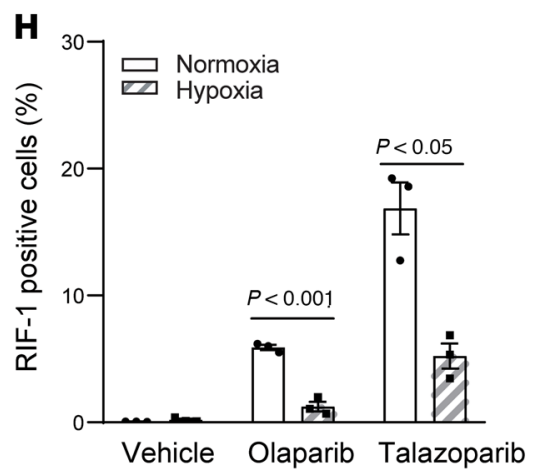

I
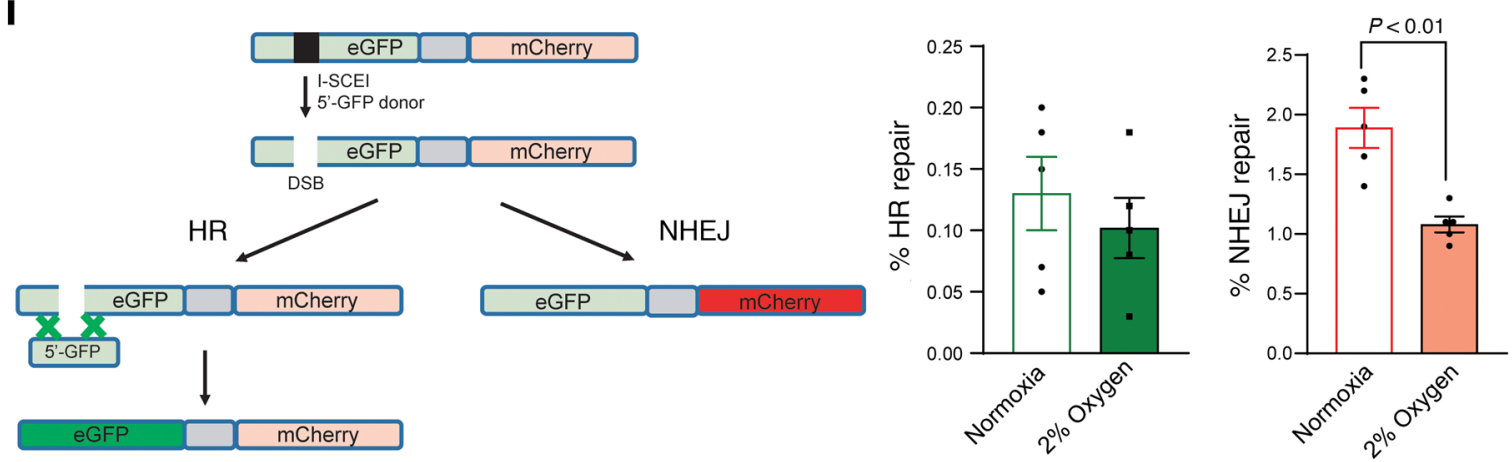
Figure 3. PARPi-induced DNA damage and activation of C-NHEJ pathway are limited in hypoxia. (A) Immunoblots of DDR proteins in SUM149 cells treated with vehicle or PARPi for 48 hours under normoxia or hypoxia. See also Supplemental Figure 3A. (B) Representative images (left) and quantification (right) of $\gamma \mathrm{H} 2 \mathrm{AX}$ foci analyzed by high-throughput microscopy in SUM149 cells after 48 hours of PARPi treatment in normoxia or hypoxia (right panel). Original magnification, $\times 20$. See also Supplemental Figure 3B. $P$ values determined by 2-way ANOVA $(n=3)$. (C) Immunoblot of chromatin-bound PARP1 and ORC2 (loading control) after 48 hours of PARPi treatment. (D-H) C-NHEJ pathway is a major contributor to PARPi toxicity in HR-deficient cells in normoxia, but not in hypoxia. (D) Average number of pDNA-PKcs (T2609) foci per cell after 48 hours of PARPi treatment $(n=$ 3); see also Supplemental Figure 3C. (E) Clonogenic survival of BRCA1deficient cells SUM149 treated for 96 hours with indicated concentrations of olaparib (left panel) or talazoparib (right panel) in combination with vehicle or $3 \mu \mathrm{M}$ DNA-PKi (NU7441) under normoxic (black lines) or hypoxic (colored lines) culture conditions, followed by 7 - to 10-day culture in the absence of inhibitor. Survival relative to untreated cells is plotted ( $n=3$ for each condition). (F) Average number of p53BP1 S25 foci per nucleus in SUM149 cells $(n=3)$; see also Supplemental Figure 3D. (C) Immunoblot of chromatin-bound p53BP1 S25 and total 53BP1 after 48 hours of PARPi treatment is shown; ORC2 is a loading control. (H) Percentage of cells with more than 3 RIF-1 foci after 48 hours of PARPi treatment. Data are represented as mean \pm SEM (represented by error bars). $P$ values determined by 2-way ANOVA. (I) Hypoxia does not reverse HR status in HR-deficient cells. The TLR system (44) was used to measure the relative ratio of end-joining (mCherry) and HR (EGFP) repair of a DSB in SUM149 cells after expression of I-Scel and 5' EGFP under normoxia and hypoxia. A diagram of the TLR is represented. $P$ values determined by 2 -tailed Student's $t$ test $(n=4$ for each condition). See also Supplemental Figure 3, E and F.

Sensitivity of HT1080 cells to talazoparib was enhanced upon knockout of Pol $\theta$ under normoxic conditions, which is consistent with previously published data confirming the role of $\operatorname{Pol} \theta$ in enhancing toxicity of PARPi (48). Interestingly, absence of Pol $\theta$ under hypoxia did not have an effect on PARPi-induced toxicity, suggesting that Poly-dependent repair is not engaged under low oxygen tensions (Figure 5D). Efficient knockout of $\operatorname{Pol} \theta$ was confirmed with measurements of mRNA levels (Figure 5E).

Combination drug administration induces synergistic cytotoxicity and decreases clonogenicity in vitro. Our findings have prompted us to hypothesize that exploiting hypoxia by inducing DNA damage might be an effective therapeutic strategy in enhancing response to PARPi. Tirapazamine (TPZ), a hypoxiaactivated prodrug, is known to cause substantially higher levels of SSBs than DSBs (52), which makes it an ideal candidate for combining with PARP inhibition.

We assessed the effect of combining TPZ with PARPi on cellular toxicity in SUM149 and OVCAR8 cells and found that this combination resulted in a significant decrease in cell survival as compared with either drug alone (Figure 6A and Supplemental Figure 5A).

Combenefit, a validated, open-access software program, was used to analyze the data and quantify possible synergistic or antagonistic drug interactions (53), and synergy scores are presented in matrix format (Figures 6B and Supplemental Figure 5B). Using a classical Lowe synergy model, we detected a substantial synergistic interaction between TPZ and PARPi, which was stronger under hypoxic conditions, with about 10-fold lower doses of TPZ required to achieve synergy scores that were similar or even higher than those under normoxic conditions. Examples are highlighted with the red squares in the matrix plots (Figure 6B). In SUM149 cells, for example, TPZ doses required to achieve a synergy score of 20 to 30 when cells are treated with $0.1 \mu \mathrm{M}$ olaparib are $1 \mu \mathrm{M}$ in hypoxia and $10 \mu \mathrm{M}$ in normoxia. A similar trend was observed with OVCAR8 cells for which a synergy score of 30 to 40 required treatment with $10 \mathrm{nM}$ talazoparib and TPZ at doses of 0.5 to $1 \mu \mathrm{M}$ in hypoxia and 5 to $10 \mu \mathrm{M}$ in normoxia. A marked increase in DNA damage (assessed by $\gamma \mathrm{H} 2 \mathrm{AX}$ foci) was observed in SUM149 with combination treatment with TPZ concentrations as low as $0.1 \mu \mathrm{M}$ in hypoxia and as high as $10 \mu \mathrm{M}$ in normoxia as compared with PARPi treatment alone. In order to achieve comparable DNA damage, $1 \mu \mathrm{M}$ and $10 \mu \mathrm{M}$ of TPZ were needed in hypoxia and normoxia, respectively (Figure 7A).

Using a relatively low concentration of TPZ $(0.1 \mu \mathrm{M})$ that is nontoxic in normoxia but moderately toxic in hypoxia, we found that SUM149 cells exhibited a significant decrease in clonogenic survival upon cotreatment with olaparib $(0.1 \mu \mathrm{M})$ or BMN673 (10 nM). OVCAR8 cells also exhibit higher sensitivity to the combination treatment of TPZ and olaparib $(1 \mu \mathrm{M})$ or TPZ and talazoparib (10 nM) as compared with either therapy alone. It is noteworthy that this effect was only observed under hypoxic conditions in both cell lines (Figures 7B and Supplemental Figure 5C) and sensitivity to the drug combination in normoxia only occurs with higher concentrations of TPZ $(1 \mu \mathrm{M})$ that are moderately toxic to the cells (Figure 7C and Supplemental Figure 5D). As shown in Figure 7D and Supplemental Figure 5E, treatment of cells with $1 \mu \mathrm{M}$ TPZ led to a significant increase (about 1.3-fold) in the generation of ROS as compared with untreated cells, whereas treatment with 0.1 $\mu \mathrm{M}$ TPZ did not result in a significant increase in the production of ROS. Since, TPZ-induced normoxic damage is caused by ROS (Supplemental Figure 5F) $(54,55)$, we conclude that, under normoxic conditions, ROS are required to cause initial DNA damage, which is normally inefficiently repaired in the presence of PARPi, leading to enhanced cell death.

HR-deficient tumor xenografts are sensitive to TPZ and PARPi combination treatment. Based on the observed synergism between TPZ and PARPi in vitro, we tested the extent to which the effect of this therapeutic combination could be found in vivo using 3 subcutaneous xenograft tumor models with different HR deficiencies. The key finding of these experiments is that TPZ selectively causes cell death in hypoxic tumor cells after its bioreductive activation (54). Tumor-bearing mice were treated with vehicle, PARPi, or a combination of PARPi and TPZ (Figure 8A). In OVCAR8 xenografts (BRCA1 functional deficiency), mice treated with low-dose olaparib $(50 \mathrm{mg} / \mathrm{kg})$ or TPZ $(20 \mathrm{mg} / \mathrm{kg})$, showed no reduction in tumor burden compared with those treated with vehicle. However, combining olaparib with TPZ resulted in a significant potentiation of PARPi cytotoxicity, as shown by the significant $(P<0.0001)$ tumor growth delay starting from day 22 of treatment when compared with vehicle, olaparib, or TPZ alone (Figure 8B). Treatment of mice with olaparib was also associated with a significant reduction in PAR levels, confirming that inhibition of PARP activity was successfully achieved in vivo (Figure $8 \mathrm{~B}$ ).

BRCA1 mutant SUM149 xenografts showed some sensitivity to talazoparib $(0.1 \mathrm{mg} / \mathrm{kg})$ and TPZ $(20 \mathrm{mg} / \mathrm{kg})$ monotherapies, while the combination yielded strong tumor-burden reduction 
A
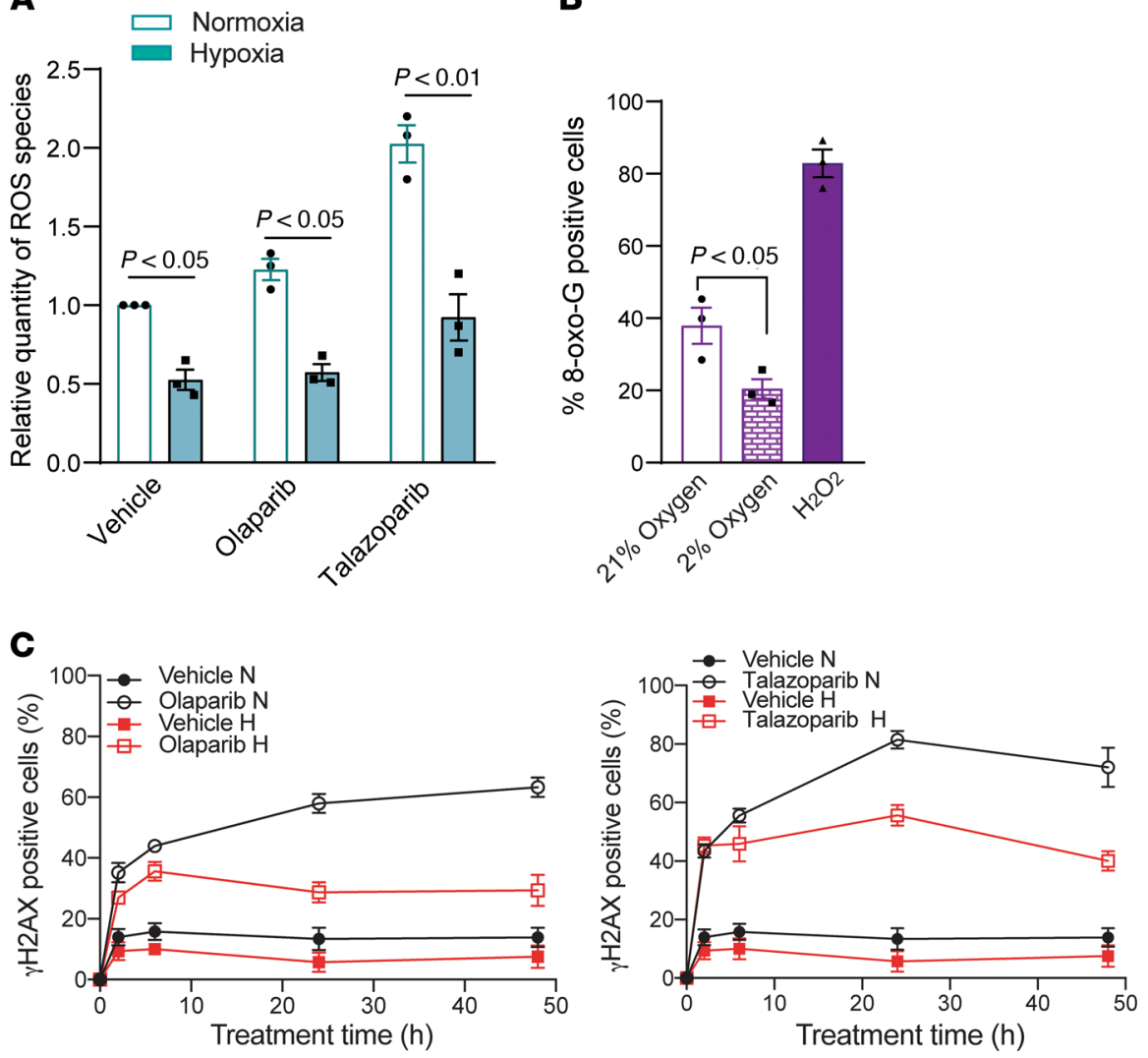

D

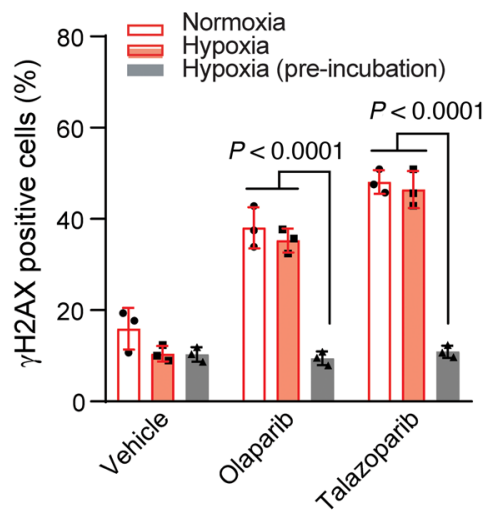

F
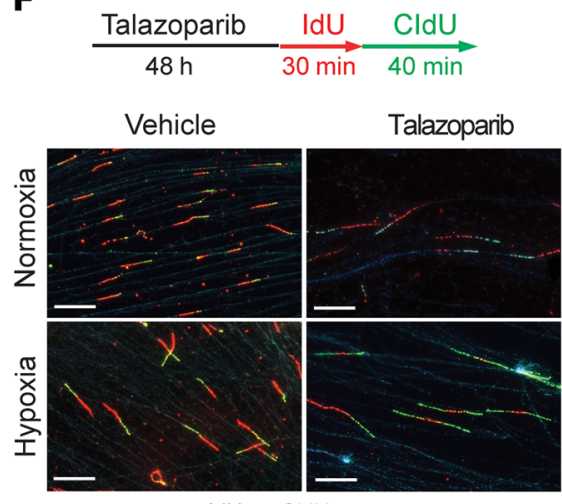

- IdU $-C l d U \_$DNA
B

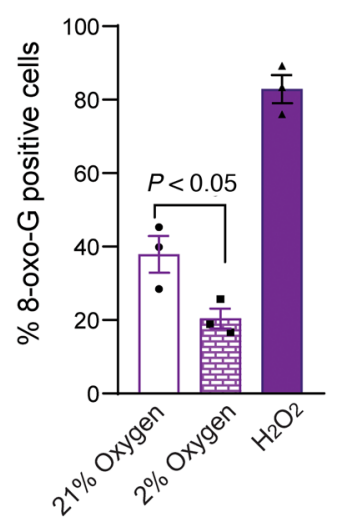

E

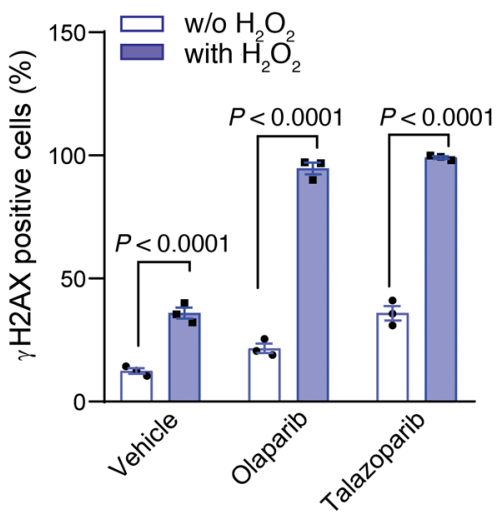

G

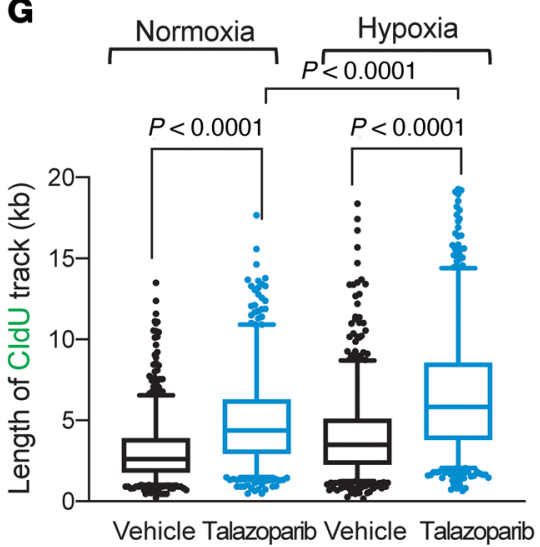

Figure 4. Hypoxia-mediated PAPRi resistance is associated with low ROS production. (A) Relative quantity of ROS (detected by DCF) produced upon treatment of cells with PAPRi for 48 hours under normoxia or hypoxia ( $P$ values determined by 2-way ANOVA, $n=3$ ). See also Supplemental Figure 4A. (B) Quantification of 8-oxo-dG staining analyzed by high-throughput microscopy in SUM149 cells after 48-hour incubation under normoxic or hypoxic culture conditions. $\mathrm{H}_{2} \mathrm{O}_{2}(50 \mu \mathrm{M})$ used as a positive control ( $P$ values determined by 2 -tailed Student's $t$ test, $n=3$ ). (C) Percentage of SUM149 cells with more than $10 \gamma \mathrm{H} 2 \mathrm{AX}$ foci per nucleus after 2 hours, 6 hours, 24 hours, and 48 hours of PARPi treatment under normoxic or hypoxic culture conditions, $n=3$. See also Supplemental Figure 4B. (D) Percentage of SUM149 cells with more than $10 \gamma \mathrm{H} 2 \mathrm{AX}$ foci per nucleus after 6 hours of PARPi treatment in normoxic or hypoxic culture conditions and after 48 hours of preincubation in hypoxia ( $P$ values determined by 2 -way ANOVA, $n=3$ ). See also Supplemental Figure 4C. (E) Percentage of SUM149 cells with more than 10 $\gamma \mathrm{H} 2 \mathrm{AX}$ foci per nucleus after 48 hours of PARPi treatment under hypoxic culture conditions followed by 2-hour treatment with $50 \mu \mathrm{M} \mathrm{H}_{2} \mathrm{O}_{2}$ ( $P$ values determined by 2-way ANOVA, $n=$ 3). (F) Experimental setup for replication fork progression assay. Representative fields of DNA fibers are indicated. Scale bars: $10 \mu \mathrm{m}$. (G) Effect of hypoxia on PARPi-induced fork regulation. DNA fibers from SUM149 cells treated with talazoparib (10nM) for 48 hours under normoxia or hypoxia. Scored forks: (normoxia) vehicle = 726; talazoparib = 633; (hypoxia) vehicle $=758$; talazoparib $=792 ; n=2$ biological replicates. Whiskers indicate the 5th and 95th percentiles, and the center values depict the median. $P$ values determined by 1 -way ANOVA.

starting from day 24 of therapy (Figure $8 \mathrm{C}$ ). Individual group comparisons showed that treatment with talazoparib had no significant effect on the growth of the SUM149 line compared with the untreated controls $\left(540 \mathrm{~mm}^{3} \pm 57.33 \mathrm{~mm}^{3}\right.$ versus $590 \mathrm{~mm}^{3} \pm 125.4 \mathrm{~mm}^{3}$; growth inhibition [GI], $8 \% ; P=0.15)$. Treatment with TPZ alone resulted in a more significant GI $\left(475 \mathrm{~mm}^{3} \pm 51.02 \mathrm{~mm}^{3}\right.$ versus $590 \mathrm{~mm}^{3}$ $\pm 125.4 \mathrm{~mm}^{3}$; GI, 20\%; $P=0.02$ ), whereas combination of talazoparib and TPZ showed the strongest treatment response $\left(254 \mathrm{~mm}^{3} \pm 12.41 \mathrm{~mm}^{3}\right.$ versus $590 \mathrm{~mm}^{3} \pm$ $125.4 \mathrm{~mm}^{3}$; GI, 58\%; $\left.P<0.0001\right)$. Tumor lysates were collected at the end of the study and used to confirm the specific ARP inhibitory effect of talazoparib therapy (Figure 8C).

We also tested HT1080 tumors, which harbor an endogenous IDH1 R132C 
mutation that confers sensitivity to PARP inhibition (13). We observed that, despite the fact that each monotherapy had no measurable effect on the growth of the tumors, combination of olaparib $(50 \mathrm{mg} / \mathrm{kg})$ and TPZ $(20 \mathrm{mg} / \mathrm{kg})$ resulted in substantial tumor growth delay from day 15 after treatment (GI, 43\%, Supplemental Figure 6A). Taken together, our data confirm that HR-deficient tumors are hypersensitive to combination treatment of PARPi and TPZ.

Overall, treatment was well tolerated, with no animal lethality. TPZ at $20 \mathrm{mg} / \mathrm{kg}$ did result in slight toxicity in this treatment regimen, with an average body weight loss of $5 \%-7 \%$. In the same experiment, animals that were treated with olaparib $(50 \mathrm{mg} / \mathrm{kg})$ or talazoparib $(0.1 \mathrm{mg} / \mathrm{kg})$ alone had maximum average body weight loss of $0.5 \%-1.5 \%$. Importantly, combination of PARPi and TPZ did not significantly increase weight loss compared with TPZ single-agent treatment in these mice (TPZ average weight loss was $5 \%$ versus combination average weight loss of $7 \% ; P=0.75$ ) (Supplemental Figure 6B). In addition, mice recovered their body weight between TPZ doses. Therefore, PARPi did not enhance the toxicity of TPZ, as judged by its minimal impact on body weight of mice.

We also performed toxicity analysis of talazoparib and TPZ treatment in the SUM149 xenograft model. Although the numbers of white blood cells, red blood cells, and platelets were reduced in the TPZ-treated group, there was no further reduction upon cotreatment with PARPi (Supplemental Figure 6C). Therefore, although TPZ did demonstrate some hematologic toxicity on its own, which is in agreement with prior preclinical investigations (56), it did not potentiate the hematologic toxicity of PARPi when the 2 agents were coadministered. Finally, histological analysis of several organs from these animals showed no histological abnormalities across treatment groups, indicating lack of gross normal tissue toxicity (Supplemental Figure 6D).

In summary, evidence for the important translational implication of our study lies in the fact that a well-tolerated combination therapy of low-dose TPZ and low-dose PARPi resulted in potent antitumor effects as compared with each monotherapy.

\section{Discussion}

PARPis play an important role in the clinical management of HR-deficient cancers. Approximately $50 \%$ of ovarian cancers and $10 \%-20 \%$ of breast, metastatic prostate, and pancreatic cancers harbor mutations in HR factors, making these tumors candidates for PARPi therapy (57). However, despite the enthusiasm for the addition of PARPis to the clinical landscape of HR-deficient tumors, not all patients benefit and many who initially respond to therapy develop resistance. Deciphering the mechanisms of resistance to PAPRis is therefore essential to improving their clinical efficacy (58). While recent studies have identified cancer cellintrinsic mechanisms driving PARPi resistance, our study reveals a mechanism by which the hypoxic tumor microenvironment promotes PARPi resistance through reduced ROS-mediated DNA damage. Tumor hypoxia is a key microenvironmental factor present in most solid tumors and is well known for its role in promoting resistance to conventional anticancer therapies (14). Taking advantage of a previously established hypoxia gene-expression signature (35) and a database of breast PDX models (34), we found that sensitivity of these tumors to olaparib was inversely correlated with the level of intratumor hypoxia.

Previous work demonstrated that the HR pathway is compromised in severe hypoxia $(19,20,33)$, resulting in synthetic lethality with PARP inhibition (21). Similarly, we found that HR is suppressed only under severe hypoxia (0.5\% oxygen) in HRproficient cells. Importantly, our study identifies a different effect under moderate hypoxia, where both HR-proficient and HRdeficient cancer cells are resistant to PARPi therapy, thereby suggesting a model where low levels of ROS under hypoxia results in reduced DNA damage (59).

We also show that hypoxia promotes resistance to inhibitors of alt-NHEJ repair in HR-deficient tumors and combining them with PAPRi had no additional benefit. In contrast, PARPi in combination with inhibitors of alt-NHEJ proteins results in increased cytotoxic DSBs and cell death under normoxic conditions $(48,51)$. Therefore, our data suggest that PARPis in combination with Pol $\theta$ inhibitors or other inhibitors of the alt-NHEJ pathways are only beneficial under normoxic conditions.

Since PARPi toxicity is dependent on accumulated DNA damage (4), one would expect that inhibition of other DNA-repair pathways would result in enhanced cell killing when combined with PARPi. For example, inhibition of the C-NHEJ pathway has previously been connected to genomic stability and reduced lethality after exposure to PARPis and chemotherapeutic agents such as platinum crosslinking agents $(36,60)$. However, combining PARPi with inhibitors of the C-NHEJ pathway, such as DNA-PKi, led to reversal of PARPi-induced lethality under both normoxic and hypoxic conditions. Since hypoxia also suppresses C-NHEJ, inhibition of DNA-PK does not provide additional advantages to hypoxic cells.

Since hypoxic cells are not inherently resistant to DNA damage, we hypothesized that agents capable of inducing single-strand DNA damage in these cells would improve their sensitivity to PARPi. We show that combining PARPi with the hypoxia-activated prodrug TPZ confers antitumor activity in HR-deficient xenografts. TPZ undergoes an initial 1-electron reduction to generate a TPZ radical. In the absence of oxygen, the radical undergoes spontaneous conversion to generate the toxic benzotriazinyl radical, causing increased DNA damage (54). In the presence of oxygen, however, the unstable radical anion is back oxidized to parental TPZ, resulting in the production of superoxide (54), which although it is not known to be highly reactive, can be converted to more toxic hydrogen peroxide and hydroxyl radicals $(55,61)$. Our data indicate that the lesions caused by these toxic molecules are not efficiently repaired in PARP-inhibited HR-deficient cells and that this damage accounts for the relatively high doses of TPZ needed to synergize with PARPi in normoxia. Therefore, TPZ can be considered a double-edged sword in elevating the concentration of ROS in normoxia and directly damaging DNA in hypoxic cells. This increased damage is further enhanced through inhibition of efficient repair by PARPi ). Despite early promise in phase II clinical trials, TPZ showed no therapeutic benefit compared with standard chemoradiotherapy or chemotherapy alone in a pivotal phase III trial (62). The failure has been attributed to poor stratification of patients based on their tumor hypoxia status (63). 
A

SUM149

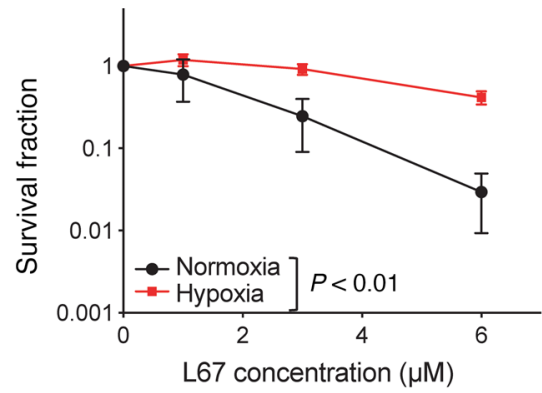

B

SUM149

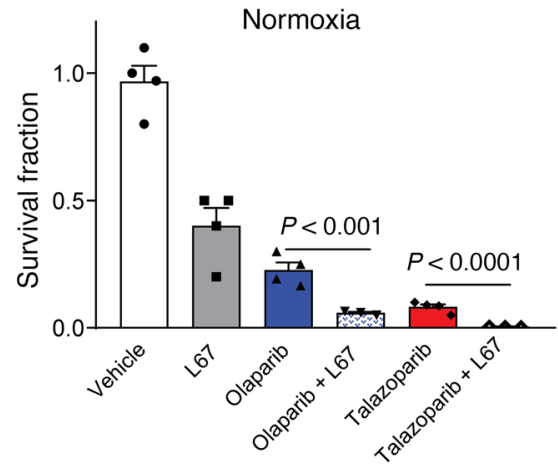

C

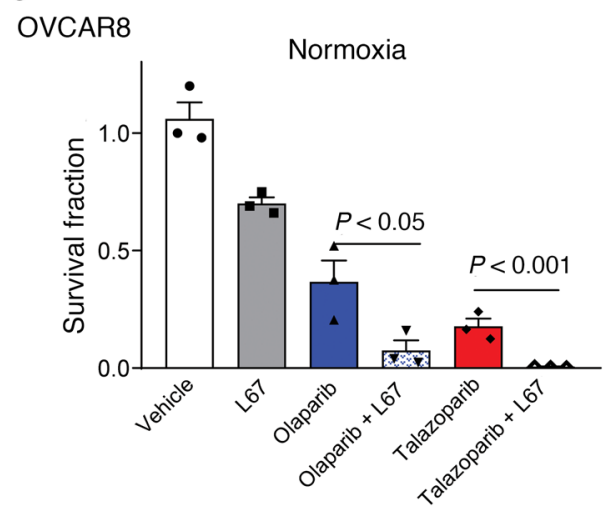

D

HT1080

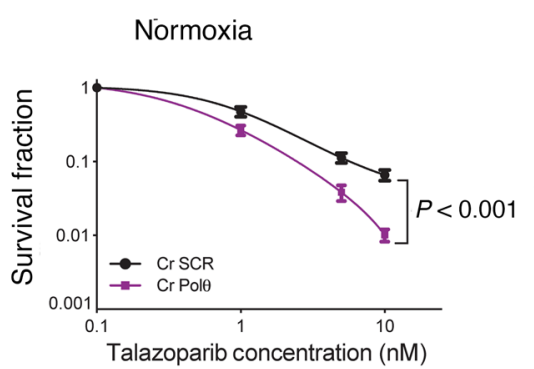

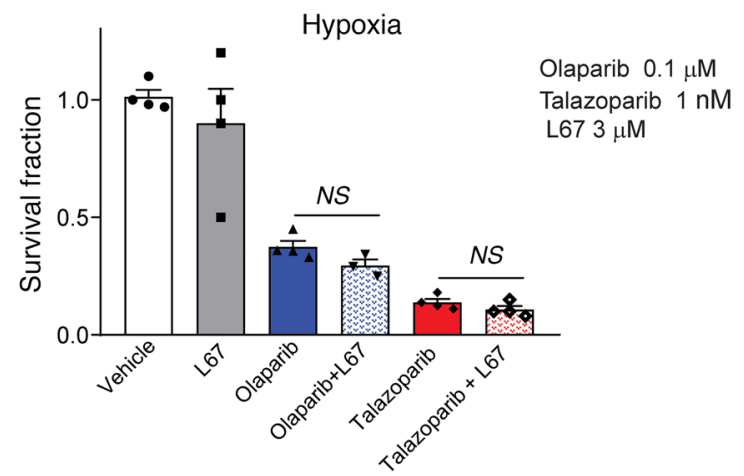

OVCAR8

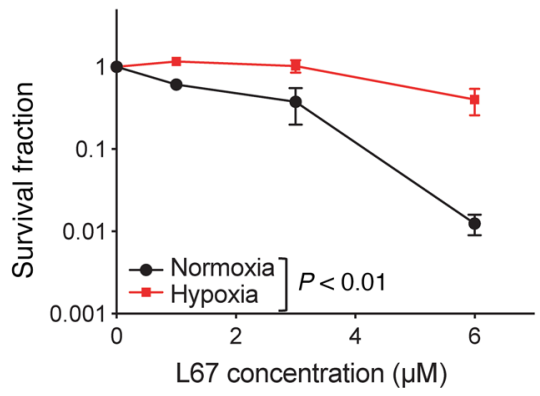

zoparib $1 \mathrm{nM}$
HT1080
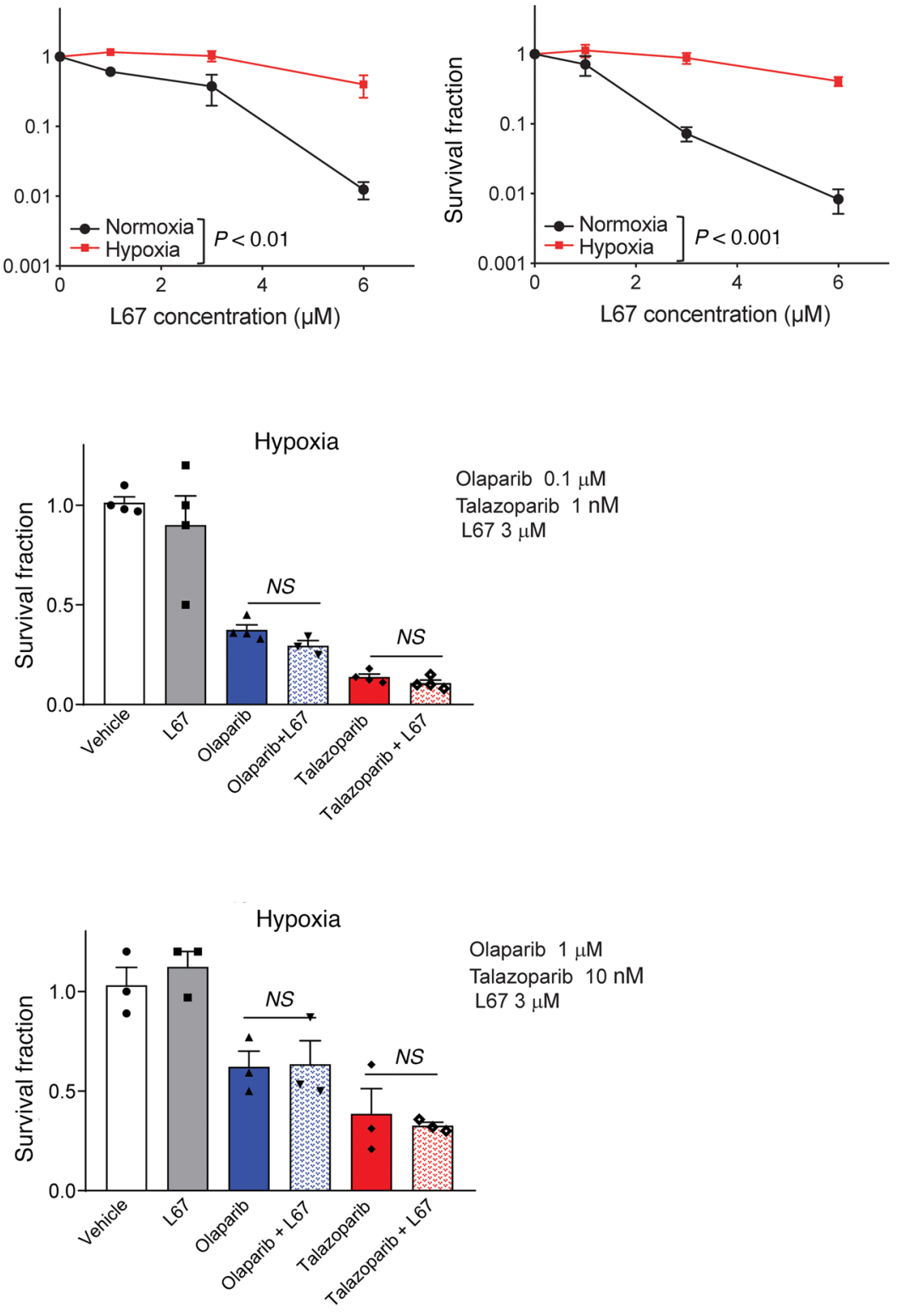

E

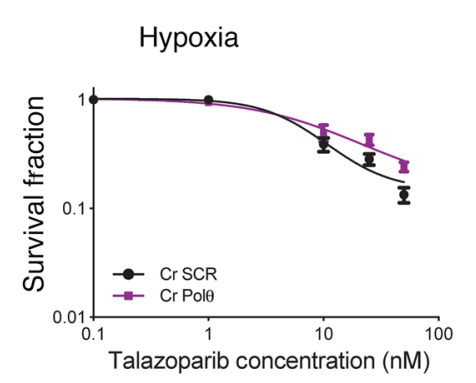


Figure 5. Hypoxia is also implicated in resistance to other inhibitors of the alt-NHEJ pathway. (A) Clonogenic formation of SUM149, OVCAR8, and HT1080 treated with indicated doses of L67 for 7 days under normoxic (black lines) or hypoxic (red lines) culture conditions, followed by 7 - to 10-day culture in the absence of inhibitor. Survival relative to vehicle-treated cells is plotted. Interaction $P$ value, normoxia versus hypoxia, determined by 2-way ANOVA. $n=3$. (B) Colony formation of SUM149 cells treated for 96 hours with $\mathrm{L} 67(3 \mu \mathrm{M})$ and olaparib $(0.1 \mu \mathrm{M})$ or talazoparib (1 $\mathrm{nM}$ ) under normoxia or hypoxia. Results expressed as survival ratio relative to vehicle-treated groups. $P$ value calculated by $t$ test, combination treatments versus single treatments. $n=4$. (C) Colony formation of OVCAR8 cells treated for 96 hours with $\mathrm{L} 67(3 \mu \mathrm{M})$ and olaparib $(1 \mu \mathrm{M})$ or talazoparib $(10 \mathrm{nM})$ under normoxia or hypoxia. Results expressed as survival ratio relative to vehicle-treated groups. $P$ value calculated by $t$ test, combination treatments versus single treatments. $n=3$. (D) Colony formation of HT1080 cells expressing the indicated CRISPR/CAS9 constructs to knockdown Pol $\theta$ and treated for 7 days with the indicated doses of talazoparib under normoxic (left panel) or hypoxic (right panel) culture conditions, followed by 7 - to 10 -day culture in the absence of inhibitor. Survival relative to vehicle-treated cells is plotted. Interaction $P$ value, normoxia versus hypoxia, determined by 2-way ANOVA. (E) qRT-PCR measuring PolQ mRNA levels in HT1080 cells. Measurements were normalized to 185 mRNA levels and expressed as fold change compared with CRISPR scrambled PolQ. $n=3$. Data are represented as mean \pm SEM (represented by error bars).

Therefore, proper stratification of patients will be necessary to ensure exploitation of the full potential of the TPZ and PARPi combination, to follow on from the success story of nimorazole, a hypoxia-targeting agent, that is currently the standard treatment for patients in Denmark receiving radiation therapy for head and neck cancer (64).

Our study reveals a new treatment combination by which targeting the hypoxic tumor microenvironment can be exploited to induce PARPi synthetic lethality. Importantly, the hypoxic cytotoxin TPZ can significantly improve PARPi efficacy without enhancing PARPi normal tissue toxicity. Therefore, we propose the evaluation of TPZ and other hypoxic cytotoxins in combination with PARPi-based therapy in future clinical trials for hypoxic tumors that are not responsive to PARPi therapy alone.

\section{Methods}

Cell culture and chemicals. OVCAR8, HT1080, CAPAN1, MDA231, and 293T cells were obtained from ATCC. SUM149 cells were a gift from James Ford (Stanford University). OVCAR8 cells were maintained in RPMI-1640 media supplemented with 10\% FBS. HT1080, MDA231, and $293 \mathrm{~T}$ cells were maintained in DMEM media supplemented with $10 \%$ FBS. CAPAN1 cells were maintained in IMDM media supplemented with 20\% FBS. SUM149 cells were cultured in Ham's F-12 supplemented with $5 \mu \mathrm{g} / \mathrm{ml}$ insulin, $1 \mu \mathrm{g} / \mathrm{ml}$ hydrocortisone, $10 \mathrm{mM}$ HEPES buffer, and 5\% FBS. All cell line media were supplemented with $1 \%$ penicillin/streptomycin (Thermo Fisher Scientific) and were cultured in a humidified chamber with $5 \% \mathrm{CO}_{2}$ at $37^{\circ} \mathrm{C}$ Hypoxic cells $\left(0.5 \%-5 \% \mathrm{O}_{2}\right)$ were maintained in a control atmosphere chamber (InVivo2 Hypoxic Workstation; Ruskinn) at $37^{\circ} \mathrm{C}$.

Olaparib (AZD2281;10mM in DMSO) was obtained from Selleckchem (catalog Ku-0059436), and talazoparib (10 mM talazoparib in DMSO) was obtained from MedChem Express (catalog HY-16106). DNA-PKi (NU7441; $10 \mathrm{mM}$ in DMSO) was from Selleckchem (cata$\log$ S2638). TPZ (10 mM in DMSO) was obtained from Sigma-Aldrich (catalog SML0552). L67 was obtained from Cayman Chemical (cat- alog 22941). $\mathrm{H}_{2} \mathrm{O}_{2}$ Solution (no. 18304) was obtained from SigmaAldrich. Enzymatic PARP activity was assessed using the Universal Chemiluminescent PARP Assay Kit (Trevigen, catalog 4676-096-K), as previously described (65).

Generation of HIF knockdown stable cells. $5 \mu \mathrm{g}$ of shRNAs against HIF1 $\alpha$, HIF2 $\alpha$, or HIF1 $\beta$ (Supplemental Table 1) were transfected into $293 \mathrm{~T}$ cells along with $2.5 \mu \mathrm{g}$ of packaging plasmids, VSVG (Addgene, catalog 8454), and delta 8.2 (Addgene, catalog 12263). Forty-eight hours after transfection, cell media containing viral particles were collected and filtered through $0.45 \mu \mathrm{m}$ filters. For infection, $2.5 \times 10^{5}$ target cells were seeded into a 6-well plate, and $1 \mathrm{ml}$ of filtered media with viral particles was mixed with $1 \mathrm{ml}$ of culture media and $5 \mu \mathrm{g} / \mathrm{ml}$ polybrene (Sigma-Aldrich, catalog H9268) and added onto target cells. Forty-eight hours after infection, infected cells were selected by incubation in media containing $2 \mu \mathrm{g} / \mathrm{ml}$ puromycin (Sigma-Aldrich, catalog P8833).

Generation of Poly CRISPR/Cas9 knockout. CRISPR/Cas9 knockouts of Pol $\theta$ were generated by transfection of $293 \mathrm{~T}$ cells with single-guide RNA (sgRNA) lentivectors targeting PolO (Abm, catalog K1682205) and the packaging plasmids VSVG (Addgene, catalog 8454) and delta 8.2 (Addgene, catalog 2263). Viral particles released into the cell-culture supernatant were filtered with $0.45 \mu \mathrm{m}$ filters. Cell lines were infected with lentivirus by 2 lentiviral infections at 24-hour intervals using polybrene, and infected cells were selected by incubation with puromycin for 3 days.

Determination of synergism. Cells were plated in 96-well plates and treated with various concentrations of drugs alone or in combination. Following treatment for 4 days in normoxia or hypoxia ( $2 \%$ oxygen), the assays were terminated using sulforhodamine B (SRB) (Sigma-Aldrich, catalog S1402) and processed as previously described (66). Briefly, cells were fixed with $50 \%$ trichloroacetic acid, followed by 30 minutes incubation at room temperature with $0.4 \%$ SRB in $1 \%$ acetic acid solution. Plates were washed and SRB solubilized with 10 $\mathrm{mM}$ Tris base $\mathrm{pH}$ 10.5. Staining intensity was read on a plate reader at $510 \mathrm{~nm}$ wavelength. Cell growth was calculated as a percentage of the control wells for each group. Synergism analysis was calculated by the Lowe model using the Combenefit software tool (53).

Colony-forming assay. Cells were plated in 6-well plates and allowed to attach for 3 hours. Cells were then treated continuously with the indicated drug concentrations for the indicated durations. At the end of the experiment, the medium was removed, and the colonies were fixed in $70 \%$ ethanol and stained with Crystal Violet (Sigma-Aldrich, catalog C3886) solution to facilitate manual counting of colonies ( $\geq 50$ cells). Clonogenic survival was calculated for each condition after correcting for plating efficiency. Survival fraction was calculated by dividing survival of each condition by survival of cells treated with vehicle only.

EdU incorporation assay and cell-cycle analysis. Cell-cycle analysis and quantification of cells in S-phase was performed using the ClickiT EdU Alexa Fluor 488 Flow Cytometry Assay Kit (Thermo Fisher Scientific, catalog C10632) according to the manufacturer's protocol. 5-Ethynyl-20-deoxyuridine (EdU) $(10 \mu \mathrm{M})$ was added to cells 2 hours before harvest. Cells were costained with propidium iodide (SigmaAldrich, catalog P4170) to quantify DNA content.

ROS quantification using DCF. Intracellular ROS production was measured by staining with dichlorodihydrofluorescein diacetate ( $\mathrm{H}_{2}$ DCFDA) (Molecular Probes, catalog MP36103). After appropriate incubations, cells were loaded with $10 \mu \mathrm{M} \mathrm{H}_{2}$ DCFDA for 30 minutes 
$\mathbf{A}$
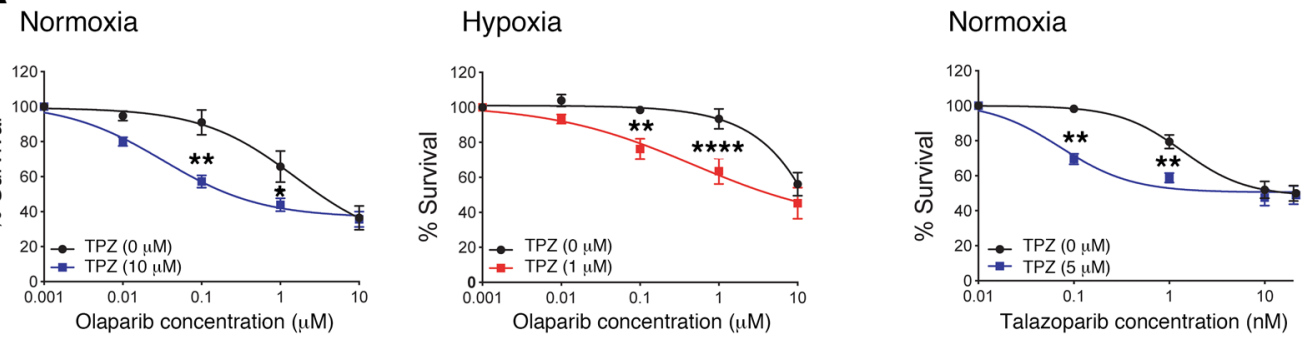

Hypoxia
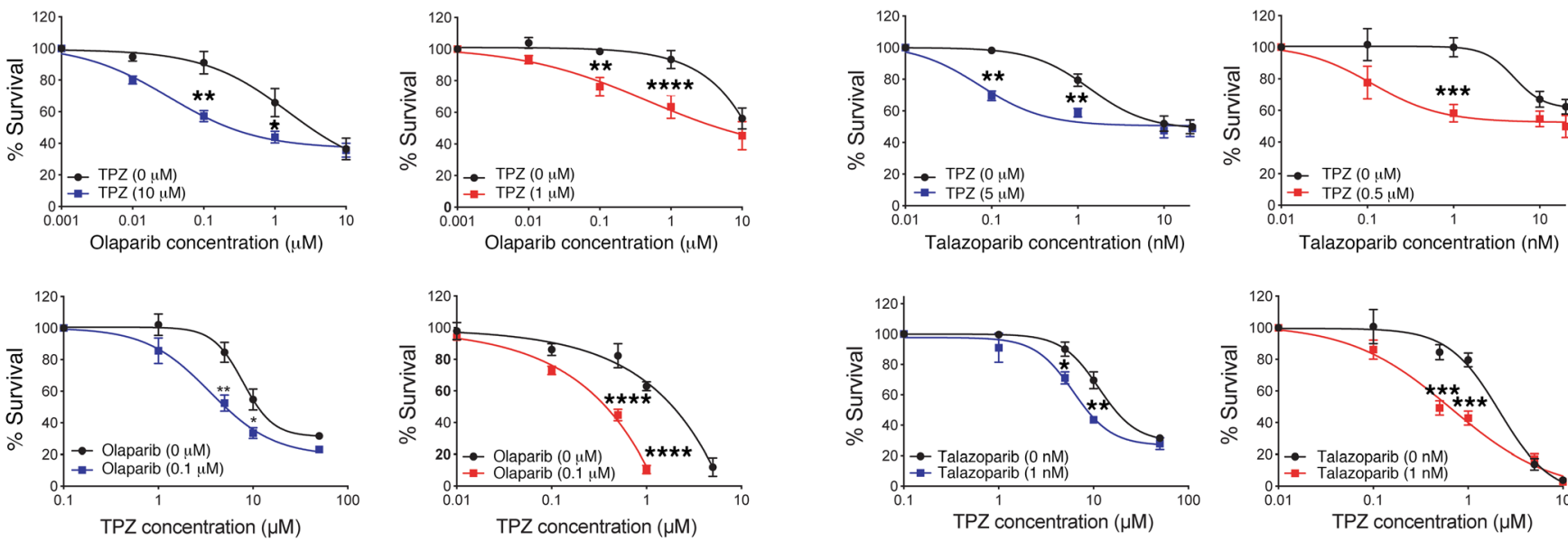

$\mathbf{B}$

Normoxia

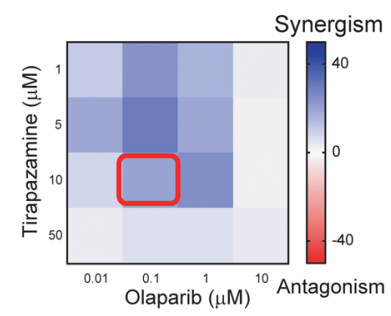

Hypoxia

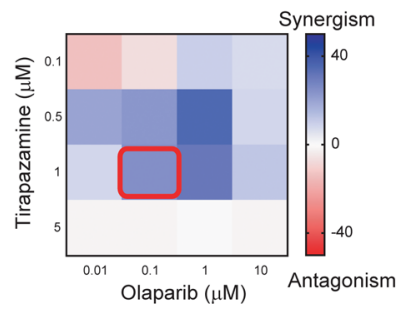

Normoxia

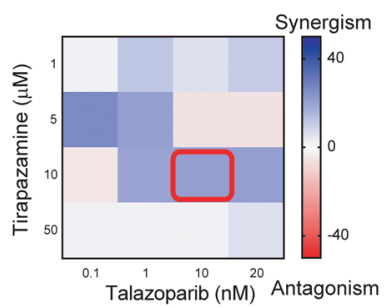

Hypoxia

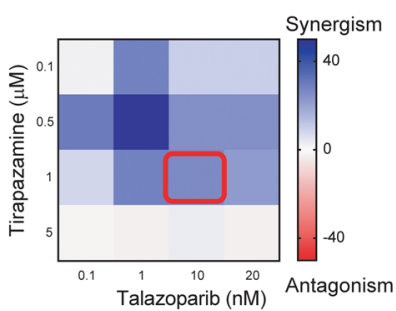

Figure 6. Combination drug administration induces synergistic cytotoxicity and decreases clonogenic survival in vitro. (A) Cell survival of SUM149 cells treated with olaparib $(0.001-10 \mu \mathrm{M})$ and TPZ $(0.1-50 \mu \mathrm{M})$ or talazoparib $(0.01-20 \mathrm{nM})$ and TPZ $(0.1-50 \mu \mathrm{M})$. Cells were treated for 4 days with the indicated drug combinations under normoxia or hypoxia, followed by SRB assay to determine cytotoxicity. $n=3$. $P$ values were determined by $t$ test, combination treatments versus single treatments. See also Supplemental Figure 5A. Data are represented as mean \pm SEM (represented by error bars). ${ }^{*} P<0.05$; ${ }^{* *} P<$ $0.01 ;{ }^{* *} P<0.001 ;{ }^{* * *} P<0.0001$. (B) HSA synergism analysis of SUM149 cells treated with varying doses of TPZ and olaparib or TPZ and talazoparib. See also Supplemental Figure 5B.

at room temperature, washed in PBS, and incubated with fresh media without $\mathrm{H}_{2}$ DCFDA for 30 minutes. DCF fluorescence was quantified by flow cytometry.

Subcellular fractionation. Subcellular fractionation was carried out with modifications, as previously reported (67). Cells were initially lysed in hypotonic buffer (10 mM HEPES, pH 7.9, $10 \mathrm{mM} \mathrm{KCl}$, $1.5 \mathrm{mM} \mathrm{MgCl}_{2}$ ), Pierce protease inhibitor mini tablets (Thermo Fisher, catalog A32955), and phosphatase inhibitor (Roche, catalog 4906845001).

The lysate was homogenized 10 times using a dounce homogenizer (pestle B) (Kimble Chase). The nuclei were then pelleted by centrifugation at $15,000 \mathrm{~g}$ at $4^{\circ} \mathrm{C}$ for 5 minutes, and lysed in high salt buffer (20 mM HEPES, pH 7.9, 0.42 M NaCl, 25\% glycerol, 1.5 mM MgCl2, $0.1 \%$ Nonidet P-40 [NP-40], 0.2 mM EDTA, Pierce protease inhibitor mini tablets [Thermo Fisher, catalog A32955], phosphatase inhibitor [Roche, catalog 4906845001]). Lysates were then homogenized 20 times using a dounce homogenizer (pestle B), and centrifuged at $15,000 \mathrm{~g}$ at $4^{\circ} \mathrm{C}$ for 15 minutes. The final pellet containing the chromatin fraction was lysed in RIPA buffer $(50 \mathrm{mM}$ Tris$\mathrm{HCl}$, pH 8.0, $150 \mathrm{mM} \mathrm{NaCl}, 5 \mathrm{mM} \mathrm{CaCl}$, 1\% NP-40, 0.5\% sodium deoxycholate, $0.1 \%$ SDS, Pierce protease inhibitor mini tablets [Thermo Fisher, catalog A32955], phosphatase inhibitor
[Roche, catalog 4906845001]) and incubated with micrococcal nuclease (Thermo Fisher, catalog 88216) at room temperature for 15 minutes to release chromatin-bound proteins. Protein binding in chromatin was assessed by immunoblotting as described below.

Protein extraction and Western blotting analysis. Cells in culture were washed with ice-cold PBS twice to completely remove residual medium. Cells were lysed with RIPA lysis buffer supplemented with Protease Inhibitor (EDTA-free) Cocktail (Roche, catalog 11836170001) to extract protein. After thorough mixing and incubation at $4^{\circ} \mathrm{C}$ for 10 minutes, lysates were centrifuged at $15,000 \mathrm{~g}$ at $4^{\circ} \mathrm{C}$ for 10 minutes, and supernatants were collected. Protein concentrations were measured using Pierce BCA Protein Assay Kit (Thermo Fisher Scientific, catalog 23225) with bovine serum albumin as a protein standard. Equal amounts of protein samples were subjected to $4 \%-12 \%$ gradient gel SDS-PAGE and electro-transferred onto PVDF membranes (Bio-Rad, catalog 1620177). After blocking with 5\% BSA in TBST (TBS with 0.1\% Tween), the membranes were incubated with the appropriate antibodies at $4^{\circ} \mathrm{C}$ overnight, followed by HRP-conjugated secondary antibodies (anti-rabbit, catalog sc2004; anti-mouse, catalog sc2354; anti-rat, catalog sc2006; antigoat, catalog sc2350; Santa Cruz Biotechnology Inc.) for 1 hour at room temperature. Proteins were detected by chemiluminescent signal using Clarity Western ECL Substrate (Bio-Rad, catalog 170-5060). 
A

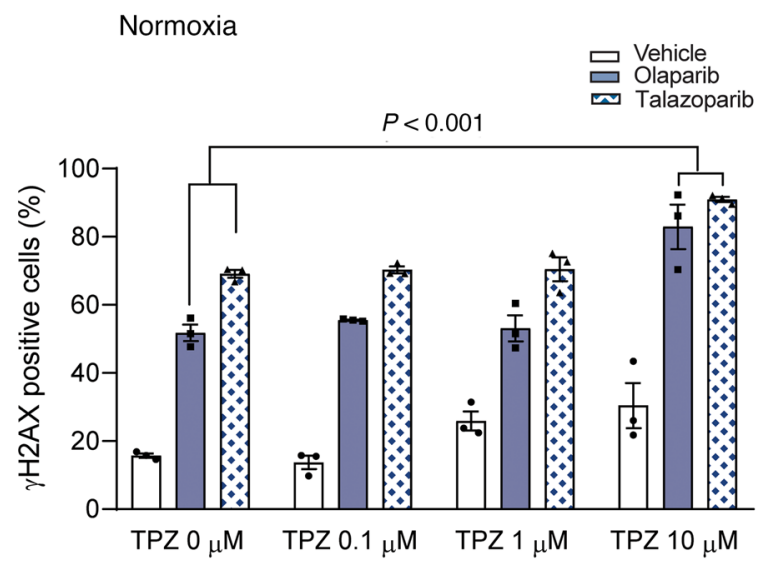

Hypoxia
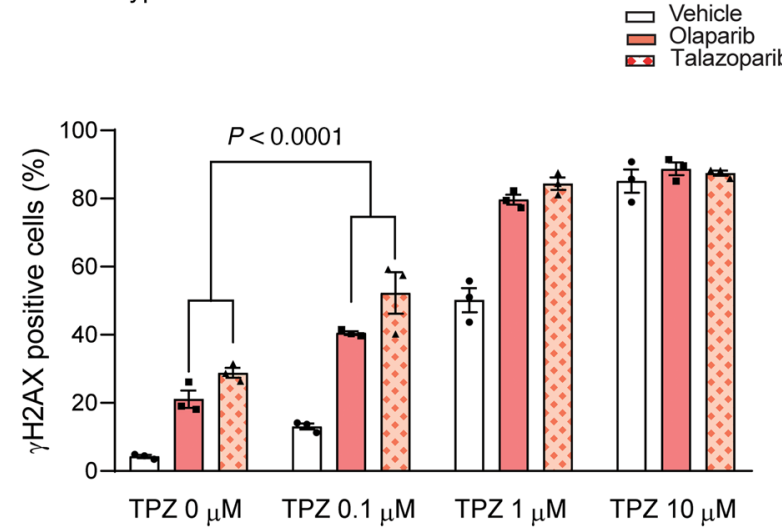

B
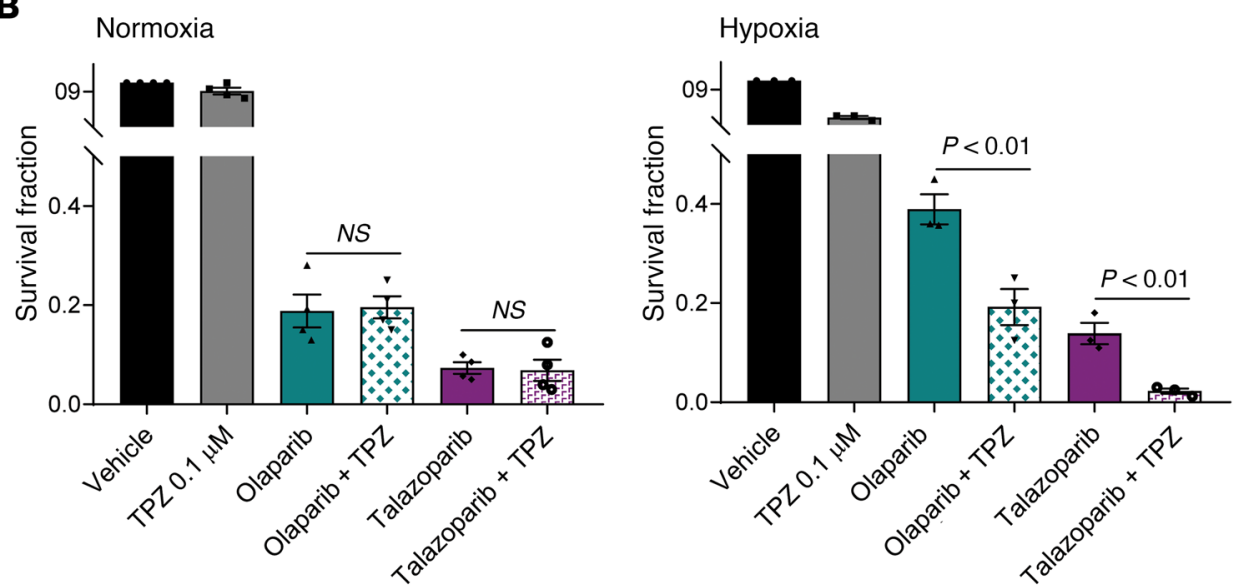

C

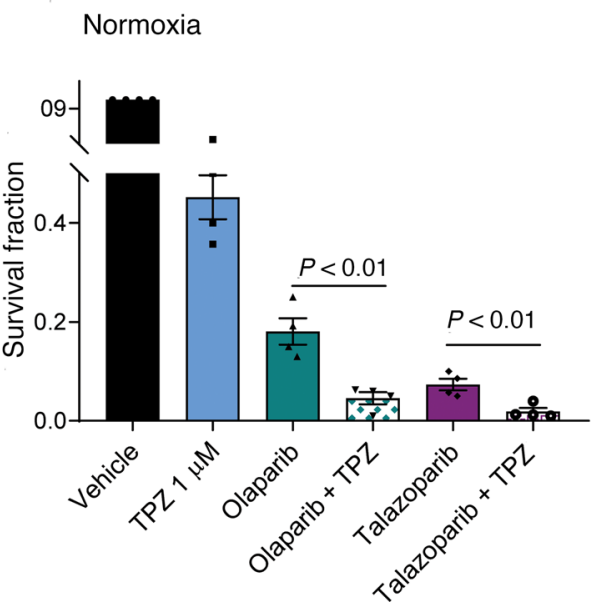

D

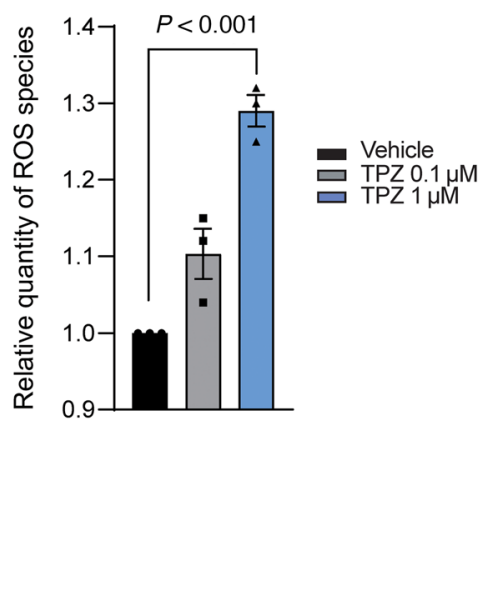

Figure 7. Combination drug administration induces DNA damage and decreases clonogenic survival in vitro. (A) Percentage of $\gamma \mathrm{H} 2 \mathrm{AX}$-positive SUM149 cells after 48-hour treatment with olaparib $(1 \mu \mathrm{M})$ and TPZ $(0.1-10 \mu \mathrm{M})$ or talazoparib $(10 \mathrm{nM})$ and TPZ (0.1-10 $\mu \mathrm{M})$ under normoxic $(n=3)$ or hypoxic $(n=$ 3) conditions. (B) Colony formation of SUM149 cells treated for 96 hours with TPZ (0.1 $\mu \mathrm{M})$ and olaparib $(0.1 \mu \mathrm{M})$ or talazoparib $(1 \mathrm{nM})$ under normoxia ( $n$ $=4$ ) or hypoxia $(n=3)$. Results expressed as survival ratio relative to vehicle-treated groups. $P$ value calculated by $t$ test, combination treatments versus single treatments. See also Supplemental Figure 5C. (C) Colony formation of SUM149 cells treated for 96 hours with TPZ ( $1 \mu \mathrm{M})$ and olaparib (0.1 $\mu$ M) or talazoparib $(1 \mathrm{nM})$ in normoxia, $P$ value calculated by $t$ tests, combination treatments versus single treatments $(n=4)$. See also Supplemental Figure $5 \mathrm{D}$. (D) Relative levels of ROS produced upon treatment of cells with $0.1 \mu \mathrm{M}$ and $1 \mu \mathrm{M}$ of TPZ for 96 hours. $P$ values calculated by 1-way ANOVA, TPZ treatment versus vehicle $(n=3)$. See also Supplemental Figure $5 \mathrm{E}$. Data are represented as mean \pm SEM (represented by error bars). 
A
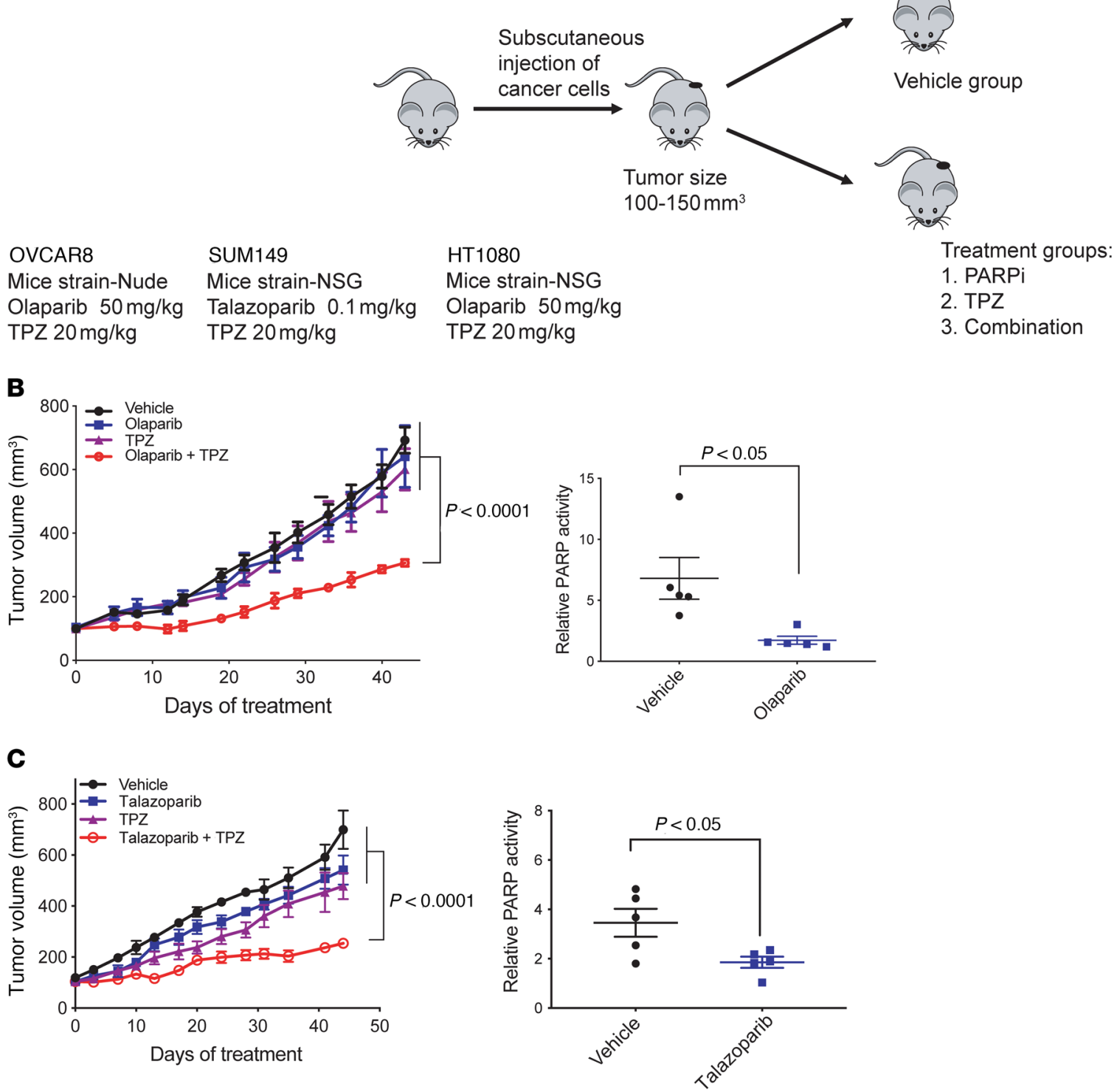

Figure 8. HR-deficient xenografts are sensitive to TPZ and PARPi combination treatment. (A) Schematic diagram of models for treatment of HR-deficient cells in vivo. Mice were injected subcutaneously with tumor cells. After tumors reached an approximate size of $100 \mathrm{~mm}^{3}$, mice were divided into vehicle and treatment groups. (B) OVCAR8 xenograft model. Left panel: growth curves of OVCAR8 tumors in control mice and mice treated with $50 \mathrm{mg} / \mathrm{kg}$ olaparib and/or $20 \mathrm{mg} / \mathrm{kg} \mathrm{TPZ}$. Two-way ANOVA: vehicle versus combination interaction, $P<0.0001$; olaparib versus combination interaction, $P<0.0001$; TPZ versus combination interaction, $P<0.0001 ; n=5$ mice in each group. Right panel: relative enzymatic PARP activity of tumor lysates collected 2 hours after the final olaparib treatment. Each dot represents data from 1 tumor. ${ }^{*} P<0.05, t$ test. See also Supplemental Figure 6A. (C) SUM149 xenograft model. Left panel: growth curves of SUM149 tumors in control mice and mice treated with $0.1 \mathrm{mg} / \mathrm{kg}$ talazoparib and/or $20 \mathrm{mg} / \mathrm{kg}$ TPZ. Vehicle versus combination: 2-way ANOVA, interaction, $P<0.0001$; talazoparib versus combination: 2 -way ANOVA, interaction, $P<0.0001$; TPZ versus combination: 2-way ANOVA, interaction, $P<0.0001 . n=5$ mice in each group. Right panel; relative enzymatic PARP activity of tumor lysates collected 2 hours after final talazoparib treatment. Each dot represents data from 1 tumor. $P<0.05$, by $t$ test. See also Supplemental Figure 6, B and C. Data are represented as mean SEM (represented by error bars).

The following primary antibodies were used to detect specific proteins: rabbit anti-p53BP1 (Ser25) (Novus, catalog N100-1803), rabbit anti-53BP1 (Novus, catalog N100-305), rabbit anti-H2AX (Abcam, catalog Ab10475), rabbit anti-PARP1 (Cell Signaling Technology, catalog 9542S), mouse anti-PAR (Trevigen, catalog NB100-122), rabbit anti-pKAP1 (S824) (Bethyl, catalog A300-767A), rabbit anti-pChk1 (S345) (Cell Signaling Technology, catalog 2348), mouse anti-Chk1 (Santa Cruz Biotechnology Inc., catalog sc-8404), mouse anti-HIF1 $\alpha$ (BD Transduction, catalog 610959), rabbit anti-HIF2 $\alpha$ (Novus, cata- $\log$ NB100-122), mouse anti-HIF $\beta$ (Novus, catalog NB100-124), goat anti-KAP1 (Bethyl, catalog A303-838A), rat anti-ORC2 (Cell Signaling Technology, catalog 4736), mouse anti- $\beta$-actin (Santa Cruz Biotechnology Inc., catalog sc47778).

RNA extraction and $q R T-P C R$. RNA was isolated from cells using RNeasy Plus Mini Kit (QIAGEN, catalog 74134) according to the manufacturer's instructions. The extracted RNA was then reverse transcribed into cDNA using the iScript cDNA Synthesis Kit (Bio-Rad, catalog 1708891) according to the manufacturer's 
instructions. Quantitative real-time PCR (qRT-PCR) was carried out using PowerUp SYBR Green Master Mix (Thermo Fisher Scientific, catalog A25742). Gene-specific primers with sequences listed in Supplemental Table 1 were used for PCR amplification. Detection and data analysis were executed with the StepOnePlus Real-Time PCR System. qRT-PCR data were normalized to $18 S$ and presented as fold changes of gene expression in the test samples compared with the control.

Immunofluorescence staining and quantitative image-based cytometry. Cells were grown in 96-well plates (Greiner Bio-One, catalog 655090) and treated with PARPi for 48 hours. Cells were washed with PBS and fixed with 4\% paraformaldehyde for 15 minutes, followed by permeabilization with $0.25 \%$ Triton $\mathrm{X}-100$ for 10 minutes. Cells were then blocked with 1\% BSA for 60 minutes and incubated with the appropriate primary antibodies overnight at $4^{\circ} \mathrm{C}$ : rabbit anti-53BP1 (Millipore, catalog PC712), rabbit anti-RIF1 (Bethyl, catalog A300568A), mouse anti-pDNAPKcs $\left(\mathrm{Thr}^{2609}\right.$ ) (Abcam, catalog ab18356), and mouse anti- gH2AX (S139) (Millipore, catalog 05-636). This was followed by secondary antibody incubation for 60 minutes at room temperature and DAPI (Sigma-Aldrich, catalog D9542) for $10 \mathrm{~min}$ utes. Cells stained for 8-oxo-dG (mouse anti-8-oxo-dG, Trevigen, catalog 4354-MC-050) were fixed in methanol for 20 minutes at $-20^{\circ} \mathrm{C}$ and stained according to the manufacturer's protocol.

Cells were washed and imaged on a fully automated ImageXpress Micro (Molecular Devices) at $\times 20$. Analysis of fluorescence intensities was performed using MetaXpress software. Intensity measurements were within a nuclear mask generated from DAPI staining. Background levels were determined for each fluorescence marker by assessing histogram plots of the signal intensities in each pixel across several images. The average background value across these images was then subtracted from the mean intensity of the given fluorescent marker in each cell. Analyses were performed by using the R2 Genomics Analysis and Visualization Platform (http://r2.amc.nl).

DNA fiber analysis. After treatment with PARPi, SUM149 cells were pulse-labeled with IdU $(50 \mu \mathrm{M})$ (Sigma-Aldrich, I7125) for 30 minutes, followed by a gentle wash with prewarmed PBS and a second pulse of CldU (400 $\mu \mathrm{M})$ (Sigma-Aldrich, C6891) for 40 minutes. Labeled cells were collected and DNA fiber spreads prepared as previously described, using 2 slides per sample (68).

DNA fibers were visualized using primary antibodies specific for IdU and CldU (BD Biosciences, catalog 347580; Abcam, catalog Ab6326, respectively) and stained with Alexa Fluor 488- or 594-conjugated secondary antibodies (Thermo Fisher). Images of well-spread DNA fibers were captured with fluorescence microscopy using an Inverted Zeiss observer $\mathrm{z} 1$ at $\times 40$ and a ZEISS Axiocam 506 mono camera (pixel size $4.54 \times 4.54 \mu \mathrm{m}$, conversion factor 0.1135). At least 15 images per sample were collected. Tracts that represent replication fork termination events (red-green-red or red-only tracts) or new origin firing during the CIdU labeling (green only) were excluded from the analysis. In all experiments, CIdU tract lengths were measured only when preceded by IdU labeling to quantify ongoing replication forks (green followed by red staining pattern). Image (NIH) was used to measure DNA fiber length. Two independent replicates were performed, and results were pooled together. Samples were collected, blinded, and spread by 2 different people so that the experiments (spreading/imaging/measuring) were performed in a totally blind manner.
TLR assay. Lentiviral constructs coding for TLR (catalog 31482) and I-Sce1 with donor e-GFP (catalog 31476) were purchased from Addgene. The plasmid was transduced by 2 lentiviral infections at 24-hour intervals using supernatants from transfected 293T cells. Cells with integrated TLR were selected with puromycin for 5 days. Cells were then transduced with I-Sce1, treated with PARPi for 48 hours in the absence of antibiotic selection, and analyzed by flow cytometry. EGFP, indicative of HR, was measured by using a 488 $\mathrm{nm}$ laser for excitation and a 510/30 filter for detection. mCherry fluorescence, which reflects NHEJ, was measured by using a $561 \mathrm{~nm}$ laser for excitation and a 610/20 filter for detection.

In vivo efficacy studies. Olaparib was solubilized in DMSO and diluted in PBS containing 10\% (w/v) 2-hydroxy-propyl-beta-cyclodextrin (Cayman Chemicals, catalog 16169). Talazoparib was prepared weekly in $10 \%$ DMAc, $6 \%$ Solutol and $84 \%$ PBS at $0.01 \mathrm{mg} / \mathrm{ml}$ and stored at $4^{\circ} \mathrm{C}$ in the dark.

Mice were maintained in a sterile environment, housed in individually vented caging systems under a 12-hour light/12-hour dark cycle, and maintained at uniform temperature and humidity. OVCAR8 xenografts were established by subcutaneous injection of $5 \times 10^{6}$ cells in serum- free medium/Matrigel mix into the mid-dorsal flank of 8- to 12-week-old female NU/NU nude mice (Charles River, catalog 088). SUM149 and HT0180 xenografts were established by injection of 3 $\times 10^{6}$ and $2 \times 10^{6}$ cells, respectively, in $0.1 \mathrm{ml}$ serum- free medium/ Matrigel mix into the middorsal flank of 8- to 12-week-old female immunocompromised Fox Chase SCID mice (Charles River, catalog 236). When tumors reached an average size of $100 \mathrm{~mm}^{3}$, mice were randomized and divided into 4 groups, according to treatments. Mice were treated with PARPi, TPZ (20 mg/kg) twice weekly by i.p. injection, the combination, or vehicle. Olaparib $(50 \mathrm{mg} / \mathrm{kg})$ was administered by i.p. injection 5 times a week. Talazoparib $(0.1 \mathrm{mg} / \mathrm{kg})$ was administered by oral gavage 5 days a week for the duration of the study. Tumor size was measured $2 \mathrm{e}$ to 3 times a week using calipers. Pimonidazole $(60 \mathrm{mg} / \mathrm{kg}$ ) (catalog HP-100, hpi) was injected i.p. 1 hour before endpoint, when animals were sacrificed following the standard protocols and tumors collected for further analysis. Organs were collected for H\&E staining, and peripheral blood was collected and analyzed with an automated analyzer using mouse specific parameters (Abaxis VetScan5).

Immunofluorescence on paraffin sections. Tissues were fixed in $10 \%$ formalin overnight and embedded in paraffin. The $5 \mu \mathrm{m}$ paraffin-embedded sections were first deparaffinized in xylene, and endogenous peroxidase was blocked by incubation with $3 \%$ hydrogen peroxide for 10 minutes. Antigen retrieval was performed by boiling the slides in EDTA buffer (10 mM, pH 6.0) in a water bath for 20 minutes. Slides were rinsed in PBS Tween $0.05 \%$ and blocked for 30 minutes with $2.5 \%$ horse serum. Slides were then incubated overnight at $4^{\circ} \mathrm{C}$ with primary antibodies (anti-pimonidazole, catalog PAb2627AP, 1:50 from hpi; anti-CA9, catalog NB100-417SS, 1:200 from Novus), followed by 1 hour with fluorescently labeled secondary antibodies at room temperature (Molecular Probes). Negative controls were treated identically, but without primary antibodies. Apoptosis was detected using the DeadEnd Fluorometric TUNEL System (Promega, catalog G3250) according to the manufacturer's instructions. Slides were mounted with fluorescence mounting medium (DAPI Fluoromount-G, SouthernBiotech, catalog 0100-20), and images were acquired using a Leica DMi8 
Fluorescent Microscope (Leica Microsystems). Colocalization of TUNEL and pimonidazole or CA9 staining was analyzed using LAS $\mathrm{X}$ software (Leica Microsystems).

Data availability. Data sets analyzed during the current study are available in TCGA (https://portal.gdc.cancer.gov/; glioblastoma, TCGA- GBM; breast, TCGA-BRCA; colorectal, TCGA-COAD; head and neck, TCGA-HNSC; liver, TCGA-LIHC; cervix, TCGA-CESC; esophagus, TCGA-ESCA; bladder, TCGA-BLCA; ovarian, TCGAOV; pancreas, TCGA-PAAD; melanoma, TCGA-SKCM; stomach, TCGA-STAD; testicular, TCGA-TGCT; thyroid, TCGA-THCA; thymoma, TCGA-THYM; cholangiocarcinoma, TCGA-CHOL) and the Breast Cancer PDTX Encyclopedia (EGAC00001000540).

Statistics. Results are presented as mean \pm SEM of at least 3 independent experiments unless otherwise stated. A $P$ value of less than 0.05 was considered statistically significant. Unless otherwise stated, significance was calculated using the 2-tailed Student's $t$ test, and correlation between groups was determined by Pearson's correlation test. Analyses were performed using GraphPad Prism 8 software. Flow cytometry data were analyzed using FlowJo software.

Study approval. Animal studies were performed in accordance with institutional guidelines and protocols approved by the Stanford University Institutional Animal Care and Use Committee.

\section{Author contributions}

MM conceived the study, performed most experiments, and wrote the manuscript. Y Xu, CGL, KNT, EJM, RKK, and Y Xiao assisted with experiments. AND assisted with analysis of animal toxicity data. JCS helped analyze high-throughput microscopy data. JB performed and analyzed the DNA fiber assay. Y Xu and JDB performed bioinformatic analysis. QTL, KAC, and EBR provided expertise and feedback. AJG conceived and coordinated the project and wrote the manuscript.

\section{Acknowledgments}

This work was supported by NIH grants CA-67166, CA-198291, and CA-197713, the Sydney Frank Foundation, and the Kimmelman Fund as well as a MRC Unit grant (to AJG) and a National Institute of Environmental Health Sciences (NIEHS) rant R01ES016486 (to KAC). We thank James Ford for providing the SUM149 cell line.

Address correspondence to: Amato Giaccia, Oxford Institute for Radiation Oncology, University of Oxford, Old Road Campus Research Building, Roosevelt Drive, Oxford OX37DQ, United Kingdom. Phone: 001.650.353.1600; Email: amato.giaccia@ oncology.ox.ac.uk.
1. Dobbelstein M, Moll U. Targeting tumoursupportive cellular machineries in anticancer drug development. Nat Rev Drug Discov. 2014;13(3):179-196.

2. Kaelin WG Jr. The concept of synthetic lethality in the context of anticancer therapy. Nat Rev Cancer. 2005;5(9):689-698.

3. Brown JS, et al. Targeting DNA repair in cancer: beyond PARP inhibitors. Cancer Discov. 2017;7(1):20-37.

4. Lord CJ, Ashworth A. PARP inhibitors: synthetic lethality in the clinic. Science. 2017;355(6330):1152-1158.

5. Eustermann S, et al. Structural basis of detection and signaling of DNA single-strand breaks by human PARP-1. Mol Cell. 2015;60(5):742-754.

6. Audebert M, et al. Involvement of poly(ADPribose) polymerase-1 and XRCC1/DNA ligase III in an alternative route for DNA double-strand breaks rejoining. J Biol Chem. 2004;279(53):55117-55126.

7. Nussenzweig A, Nussenzweig MC. A backup DNA repair pathway moves to the forefront. Cell. 2007;131(2):223-225.

8. Satoh MS, Lindahl T. Role of poly(ADPribose) formation in DNA repair. Nature. 1992;356(6367):356-358.

9. Farmer $\mathrm{H}$, et al. Targeting the DNA repair defect in BRCA mutant cells as a therapeutic strategy. Nature. 2005;434(7035):917-921.

10. Bryant HE, et al. Specific killing of BRCA2deficient tumours with inhibitors of poly(ADPribose) polymerase. Nature. 2005;434(7035):913-917.

11. D'Andrea AD. Susceptibility pathways in Fanconi's anemia and breast cancer. $N$ Engl J Med. 2010;362(20):1909-1919.

12. Murai J, et al. Trapping of PARP1 and PARP2 by clinical PARP inhibitors. Cancer Res. 2012;72(21):5588-5599.
13. Sulkowski PL, et al. 2-Hydroxyglutarate produced by neomorphic IDH mutations suppresses homologous recombination and induces PARP inhibitor sensitivity. Sci Transl Med. 2017;9(375):eaal2463.

14. Brown JM. Exploiting the hypoxic cancer cell: mechanisms and therapeutic strategies. Mol Med Today. 2000;6(4):157-162.

15. Hanahan D, Weinberg RA. Hallmarks of cancer: the next generation. Cell. 2011;144(5):646-674.

16. Hammond EM, et al. The meaning, measurement and modification of hypoxia in the laboratory and the clinic. Clin Oncol (R Coll Radiol) 2014;26(5):277-288.

17. Lu Y, et al. Hypoxia-induced epigenetic regulation and silencing of the BRCA1 promoter. $\mathrm{Mol}$ Cell Biol. 2011;31(16):3339-3350.

18. Bindra RS, et al. Hypoxia-induced down-regulation of BRCA1 expression by E2Fs. Cancer Res. 2005;65(24):11597-11604.

19. Bindra RS, et al. Down-regulation of Rad51 and decreased homologous recombination in hypoxic cancer cells. Mol Cell Biol. 2004;24(19):8504-8518.

20. Chan N, et al. Chronic hypoxia decreases synthesis of homologous recombination proteins to offset chemoresistance and radioresistance. Cancer Res. 2008;68(2):605-614.

21. Chan N, et al. Contextual synthetic lethality of cancer cell kill based on the tumor microenvironment. Cancer Res. 2010;70(20):8045-8054.

22. Hegan DC, et al. Inhibition of poly(ADP-ribose) polymerase down-regulates BRCA1 and RAD51 in a pathway mediated by E2F4 and p130. Proc Natl Acad Sci U S A. 2010;107(5):2201-2206.

23. Tutt A, et al. Oral poly(ADP-ribose) polymerase inhibitor olaparib in patients with BRCA1 or BRCA2 mutations and advanced breast cancer: a proof-of-concept trial. Lancet. 2010;376(9737):235-244.
24. Audeh MW, et al. Oral poly(ADP-ribose) polymerase inhibitor olaparib in patients with BRCA1 or BRCA2 mutations and recurrent ovarian cancer: a proof-of-concept trial. Lancet. 2010;376(9737):245-251.

25. Harvey V, et al. Phase III trial comparing three doses of docetaxel for second-line treatment of advanced breast cancer. JClin Oncol. 2006;24(31):4963-4970.

26. Gordon AN, et al. Recurrent epithelial ovarian carcinoma: a randomized phase III study of pegylated liposomal doxorubicin versus topotecan. J Clin Oncol. 2001;19(14):3312-3322.

27. Chan SL, Mok T. PARP inhibition in BRCAmutated breast and ovarian cancers. Lancet. 2010;376(9737):211-213.

28. Ame JC, et al. PARP-2, A novel mammalian DNA damage-dependent poly(ADP-ribose) polymerase. J Biol Chem. 1999;274(25):17860-17868.

29. Liu X, et al. Acquired resistance to combination treatment with temozolomide and ABT-888 is mediated by both base excision repair and homologous recombination DNA repair pathways. Mol Cancer Res. 2009;7(10):1686-1692.

30. Rottenberg S, et al. High sensitivity of BRCA1-deficient mammary tumors to the PARP inhibitor AZD2281 alone and in combination with platinum drugs. Proc Natl Acad Sci US A. 2008;105(44):17079-17084.

31. Gogola E, et al. Selective loss of PARG restores PARylation and counteracts PARP inhibitormediated synthetic lethality. Cancer Cell. 2018;33(6):1078-1093.

32. Pettitt SJ, et al. A genetic screen using the PiggyBac transposon in haploid cells identifies Parp1 as a mediator of olaparib toxicity. PLoS One. 2013;8(4):e61520.

33. Meng AX, et al. Hypoxia down-regulates DNA double strand break repair gene expression in prostate cancer cells. Radiother Oncol. 
2005;76(2):168-176.

34. Bruna A, et al. A biobank of breast cancer explants with preserved intra-tumor heterogeneity to screen anticancer compounds. Cell. 2016;167(1):260-274.

35. Buffa FM, et al. Large meta-analysis of multiple cancers reveals a common, compact and highly prognostic hypoxia metagene. Br J Cancer. 2010;102(2):428-435.

36. Patel AG, et al. Nonhomologous end joining drives poly(ADP-ribose) polymerase (PARP) inhibitor lethality in homologous recombination-deficient cells. Proc Natl Acad Sci U S A. 2011;108(8):3406-3411.

37. Chan DW, et al. Autophosphorylation of the DNA-dependent protein kinase catalytic subunit is required for rejoining of DNA double-strand breaks. Genes Dev. 2002;16(18):2333-2338.

38. Callen E, et al. 53BP1 mediates productive and mutagenic DNA repair through distinct phosphoprotein interactions. Cell. 2013;153(6):1266-1280.

39. Zimmermann M, et al. 53BP1 regulates DSB repair using Rif1 to control 5' end resection. Science. 2013;339(6120):700-704.

40. Di Virgilio M, et al. Rif1 prevents resection of DNA breaks and promotes immunoglobulin class switching. Science (New York, NY). 2013;339(6120):711-715.

41. Bouwman P, et al. 53BP1 loss rescues BRCA1 deficiency and is associated with triple-negative and BRCA-mutated breast cancers. Nat Struct Mol Biol. 2010;17(6):688-695.

42. Chapman JR, et al. RIF1 is essential for 53BP1dependent nonhomologous end joining and suppression of DNA double-strand break resection. Mol Cell. 2013;49(5):858-871.

43. Bunting SF, et al. 53BP1 inhibits homologous recombination in Brca1-deficient cells by blocking resection of DNA breaks. Cell. 2010;141(2):243-254.

44. Certo MT, et al. Tracking genome engineering outcome at individual DNA breakpoints. Nat Methods. 2011;8(8):671-676.

45. Yu TW, Anderson D. Reactive oxygen speciesinduced DNA damage and its modification: a chemical investigation. Mutat Res. 1997;379(2):201-210.

46. Zeman MK, Cimprich KA. Causes and consequences of replication stress. Nat Cell Biol. 2014;16(1):2-9.

47. Maya-Mendoza A, et al. High speed of fork progression induces DNA replication stress and genomic instability. Nature. 2018;559(7713):279-284.

48. Ceccaldi R, et al. Homologous-recombination-deficient tumours are dependent on Pol $\theta$-mediated repair. Nature. 2015;518(7538):258-262.

49. Mateos-Gomez PA, et al. Mammalian polymerase $\theta$ promotes alternative NHEJ and suppresses recombination. Nature. 2015;518(7538):254-257.

50. Newman EA, et al. Alternative NHEJ pathway components are therapeutic targets in high-risk neuroblastoma. Mol Cancer Res. 2015;13(3):470-482.

51. Tobin LA, et al. Targeting abnormal DNA double-strand break repair in tyrosine kinase inhibitor-resistant chronic myeloid leukemias. Oncogene. 2013;32(14):1784-1793.

52. Jones GD, Weinfeld M. Dual action of tirapazamine in the induction of DNA strand breaks. Cancer Res. 1996;56(7):1584-1590.

53. Di Veroli GY, et al. Combenefit: an interactive platform for the analysis and visualization of drug combinations. Bioinformatics. 2016;32(18):2866-2868.

54. Siim BG, et al. Selective potentiation of the hypoxic cytotoxicity of tirapazamine by its 1-N-oxide metabolite SR 4317. Cancer Res. 2004;64(2):736-742.

55. Moriwaki T, et al. Cytotoxicity of tirapazamine (3-Amino-1,2,4-benzotriazine-1,4-dioxide)induced DNA damage in chicken DT40 cells. Chem Res Toxicol. 2017;30(2):699-704.

56. Siemann DW, Hinchman CA. Potentiation of cisplatin activity by the bioreductive agent tirapazamine. Radiother Oncol. 1998;47(2):215-220.

57. Lord CJ, Ashworth A. BRCAness revisited. Nat Rev Cancer. 2016;16(2):110-120.

58. D'Andrea AD. Mechanisms of PARP inhibitor sensitivity and resistance. DNA Repair (Amst). 2018;71:172-176.

59. Jagannathan L, et al. Oxidative stress under ambient and physiological oxygen tension in tissue culture. Curr Pharmacol Rep. 2016;2(2):64-72.

60. Adamo A, et al. Preventing nonhomologous end joining suppresses DNA repair defects of Fanconi anemia. Mol Cell. 2010;39(1):25-35.

61. Dunford HB. Free radicals in iron-containing systems. Free Radic Biol Med. 1987;3(6):405-421.

62. Reddy SB, Williamson SK. Tirapazamine: a novel agent targeting hypoxic tumor cells. Expert Opin Investig Drugs. 2009;18(1):77-87.

63. Spiegelberg L, et al. Hypoxia-activated prodrugs and (lack of) clinical progress: the need for hypoxia-based biomarker patient selection in phase III clinical trials. Clin Transl Radiat Oncol. 2019;15:62-69.

64. Wardman P. Chemical radiosensitizers for use in radiotherapy. Clin Oncol ( $R$ Coll Radiol). 2007;19(6):397-417.

65. Liu SK, et al. A novel poly(ADP-ribose) polymerase inhibitor, ABT-888, radiosensitizes malignant human cell lines under hypoxia. Radiother Oncol. 2008;88(2):258-268.

66. Vichai V, Kirtikara K. Sulforhodamine B colorimetric assay for cytotoxicity screening. Nat Protoc. 2006;1(3):1112-1116.

67. Guo R, et al. Identification and analysis of new proteins involved in the DNA damage response network of Fanconi anemia and Bloom syndrome. Methods. 2009;48(1):72-79.

68. Jackson DA, Pombo A. Replicon clusters are stable units of chromosome structure: evidence that nuclear organization contributes to the efficient activation and propagation of $S$ phase in human cells. JCell Biol. 1998;140(6):1285-1295. 